\title{
Simple and minimally invasive SID devices for native mass spectrometry
}

Dalton T. Snyder ${ }^{1}$; Erin Panczyk ${ }^{1,2}$; Arpad Somogyi ${ }^{1,3}$; Desmond Kaplan ${ }^{4}$; Vicki Wysocki*1-3

${ }^{1}$ Resource for Native MS Guided Structural Biology

${ }^{2}$ Department of Chemistry and Biochemistry

${ }^{3}$ Campus Chemical Instrument Center, Mass Spectrometry and Proteomics

The Ohio State University, Columbus OH, USA 43210

${ }^{4}$ KapScience LLC, Tewksbury, MA, USA 01876

*Corresponding author: wysocki.11@osu.edu

\section{Graphical Abstract}

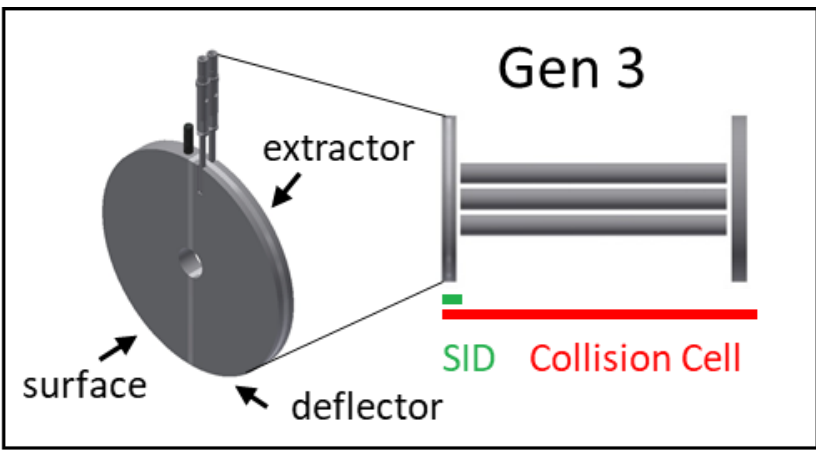

\section{Abstract}

We describe a set of simple devices for surface-induced dissociation of protein complexes on three instrument platforms. All of the devices use a novel yet simple split lens geometry that is minimally invasive (requiring a few $\mathrm{mm}$ along the ion path axis) and easier to operate than prior generations of devices. The split lens is designed to be small enough to replace the entrance lens of a Bruker FT-ICR collision cell, the dynamic range enhancement (DRE) lens of a Waters Q-IM-TOF, or the exit lens of a transfer multipole of a Thermo Scientific Extended Mass Range (EMR) Orbitrap. Despite the decrease in size and reduction in number of electrodes to 3 (from 10-12 in Gen 1 and 6 in Gen 2), we show sensitivity improvement in a variety of cases across all platforms while also maintaining SID capabilities across a wide mass and energy range. The coupling of SID, high resolution, and ion mobility is demonstrated for a variety of protein complexes of varying topologies.

\section{Keywords}

surface-induced dissociation; protein complex; native mass spectrometry; split lens

\section{Introduction}


Surface-induced dissociation (SID) as an analytical technique dates back to landmark 1975 and 1985 papers from Cooks' laboratory. ${ }^{1,2}$ SID was originally studied as an alternative activation method to the more commonly employed collision-induced dissociation (CID), with $d_{3}$-acetophenone and ethylphenyl ether as the initial case studies. Although fragment ions of the same $m / z$ were produced by SID and CID, greater energy deposition was noted for the former, giving rise to measurable differences in relative abundances of fragment ions. Other key features of SID are narrow internal energy distributions compared to $\mathrm{CID}^{3}$ and less ion restructuring (particularly of proteins and protein complexes) which is presumably due to the single-collision, high energy input nature of SID. ${ }^{4}$

Although early SID development and applications involved small molecular cations and peptide fragmentation models, ${ }^{5-8}$ protein complexes have emerged over the past decade as promising analytes for interrogation. Native protein complexes are particularly difficult to probe by mass spectrometry. Because they are in low charge states and typically have more folded structures than denatured proteins, they generally resist electron-based fragmentation methods (electron transfer dissociation, electron capture dissociation, and electron ionization dissociation), ${ }^{9-11}$ and in cases where fragments are observed, they are typically formed from surface-exposed residues of the native complex or from the restructured, non-native complex after collisional activation. ${ }^{12,13}$ Moreover, these methods generally do not produce subcomplexes from intact macromolecular species and so are of limited utility for deducing quaternary structure. Collision-induced dissociation of protein complexes produces restructured monomer in many cases and ( $\mathrm{n}-1)$ mer with asymmetric charge partitioning but does not directly generate subcomplexes consistent with intersubunit connectivity. ${ }^{14-16}$ Ultraviolet photodissociation ${ }^{17}$ can result in production of subcomplexes in some cases, ${ }^{18}$ but like infrared multiphoton dissociation ${ }^{19}$ it is usually used to produce extensive sequence fragments. Surface-induced dissociation can create both sequence fragments (usually b/y ions from peptides) and subcomplexes (from protein complexes) that are consistent with protein quaternary structure. ${ }^{4,20-22}$ Moreover, SID can be utilized to deduce a wide array of protein complex characteristics including topology, ${ }^{23,24}$ intersubunit interaction strength ${ }^{25}$ and ligand binding. ${ }^{26}$

Despite nearly 35 years of SID development from a variety of research groups, SID is not yet a commercialized or widely available technique, in contrast to CID. Its applicability to protein complexes ${ }^{4,21,22,27}$ has only recently been possible due to improvements in instrumentation for native MS. ${ }^{14,28-31}$ Many SID device designs have been tested over the years. SID can be implemented in triple quadrupole geometries in a variety of ways, ${ }^{32}$ with the quadrupoles mounted either orthogonally or collinearly and a surface placed in between. ${ }^{33,34}$ SID can be accomplished in a quadrupole ion trap by pulsing the endcaps to a high potential, causing the ions to collide with the ring electrode. ${ }^{35}$ Surfaces can be placed inside electrostatic linear ion traps ${ }^{36}$ or reflectrons ${ }^{37}$ in order to cause collisions. In FT-ICR instruments, ions can be made to collide with a surface on a direct-insertion probe placed inside the FTICR cell, ${ }^{38,39}$ a mesh electrode substituted for the front endcap, ${ }^{40}$ or the rear endcap of the FT-ICR cell itself. ${ }^{41}$ Notably, none of these configurations have been tested with protein complexes and so their applicability to macromolecular species remains unclear. ${ }^{28}$

A 10-lens in-line SID device (termed 'Gen 1' in this paper, Figure 1) was built and installed in several Waters Synapt Q-IM-TOF platforms in our laboratory ${ }^{27}$ and has been successfully used for fragmentation of protein complexes over a wide mass and energy range. This design was then translated to Thermo Scientific Extended Mass Range (EMR) Orbitrap ${ }^{42}$ and Bruker FT-ICR ${ }^{43}$ platforms (also termed 'Gen 1' devices) and subsequently simplified to 6-lens systems with tilted surfaces as a second 
generation ('Gen 2 ') of devices in the Synapt ${ }^{44}$ and FT-ICR. ${ }^{45}$ Nonetheless, there is still a desire to simplify and minimize SID device design further while also improving sensitivity.

In this work, we describe simple and minimally invasive devices for surface-induced dissociation of protein complexes across multiple instrument platforms, namely a Bruker solariX XR FT-ICR, Waters Synapt G1 and G2 Q-IM-TOF mass spectrometers, and a Thermo Scientific Extended Mass Range (EMR) Orbitrap. The SID device across all platforms is a simple yet novel doubly-split lens that can replace the entrance lens of a multipole, quadrupole, or collision cell or can replace split lenses used for ion gating or ion beam attenuation.

\section{Experimental}

\section{Chemicals}

Ammonium acetate, triethylammonium acetate (TEAA), ethylenediamine diacetate (EDDA), perfluoroheptanoic acid (PFHA), cesium bicarbonate, cesium iodide, and glutamate dehydrogenase (GDH) were purchased from Sigma-Aldrich (St. Louis, MO). Pyruvate kinase from rabbit and C-reactive protein were purchased from Lee Biosolutions (Maryland Heights, MO, USA). HFQ65, HFQ102, toyocamycin nitrile hydratase (TNH), and Trp RNA binding attenuation protein (TRAP) were obtained from collaborator laboratories (HFQ65 and HFQ102 from the Woodson group, Johns Hopkins University; TNH from the Bandarian group, The University of Utah; and TRAP from the Foster group, The Ohio State University and the Gollnick group, State University of NY, Buffalo). Protein complexes were buffer exchanged twice into $200 \mathrm{mM}$ ammonium acetate using size exclusion chromatography spin columns (Bio-Rad) with a $6 \mathrm{kDa}$ cutoff and further diluted in $200 \mathrm{mM}$ ammonium acetate to 5-10 $\mu \mathrm{M}$ concentration of protein complex. TEAA and EDDA were used as charge reducing agents and were added to protein solutions at a final concentration of $60 \mathrm{mM}$ reducing agent and $140 \mathrm{mM}$ ammonium acetate. Perfluoroheptanoic acid (PFHA) and cesium bicarbonate were used for mass calibration on the FT-ICR (CsI was used on the other platforms) and prepared together at a $10 \mathrm{mM}$ working concentration in water.

\section{Ionization}

Nanoelectrospray ionization at $\sim 1 \mathrm{kV}$ was used to generate ions of native protein complexes for analysis. Borosilicate nanospray capillaries with $\sim 2 \mu \mathrm{m}$ tip diameter were prepared in house using a Sutter Instrument P-97 tip puller (Novato, CA).

\section{Instrumentation}

A Bruker solariX XR 15 T FT-ICR mass spectrometer equipped with a nanoelectrospray ionization source was used for part of this work. All rf devices were set to their highest voltages and lowest frequencies, aside from the transport multipole between the collision cell and the FT-ICR cell, which was set at $2 \mathrm{MHz}$. The collision cell was filled with argon for collisional cooling and ion activation. Most spectra were acquired as an average of 10 scans (unless otherwise noted), generally with a mass range of $\mathrm{m} / \mathrm{z} 1,000$ to $\mathrm{m} / \mathrm{z} 20,000,1 \mathrm{M}$ transient length ( $2.2 \mathrm{~s}$ ), and time-of-flight in the range of 1.5-2 ms, depending on the $m / z$ range of the analyte ions. Higher-resolution spectra were acquired with $4 \mathrm{M}$ transient length and low $m / z$ of 1,000 ( $\sim 9$ s transient). 
Experiments were also conducted on Waters Synapt G1 and Synapt G2 Q-IM-TOF platforms equipped with a nanoelectrospray ionization source. Typical settings for TOF mode (no ion mobility) were as follows: capillary voltage, $700 \mathrm{~V}$; source temperature, $20^{\circ} \mathrm{C}$; sampling cone, 40 (arb. units); extraction cone, 1.0 (arb. units); backing pressure, 4-8 mbar (depending on the size of the protein complex); trap pressure, 1.13e-2 mbar; trap CE, 4 (arb. units); transfer CE, 4 (arb. units); ion energy (resolving quadrupole), 0.5 (arb. units); trap entrance DC, 2.0 (arb. units); trap bias, 10 (arb. units); trap exit, 5 (arb. units). Experiments were also conducted in mobility mode with the IM gas flow generally set to $20 \mathrm{~mL} / \mathrm{min}(\mathrm{G} 1)$ and $60 \mathrm{~mL} / \mathrm{min}(\mathrm{G} 2)$.

A third instrument platform we modified was a Thermo Scientific EMR Orbitrap (modified Exactive Plus) that was previously modified inhouse by the addition of a selection quadrupole and our traditional 'Gen 1' SID device. ${ }^{42}$ Typical settings are as follows: resolution setting, 35k (128 ms transient); trap gas, 5 (10 for glutamate dehydrogenase and GroEL); rf voltages set to maximum; source voltage, 1 $\mathrm{kV}$; source temperature, $200^{\circ} \mathrm{C}$; source DC offset, $7 \mathrm{~V}$; injection flatapole, $7 \mathrm{~V}$; interflatapole lens, $6 \mathrm{~V}$; bent flatapole offset, $5 \mathrm{~V}$; transfer guide offset, $5 \mathrm{~V}$; HCD mode, on (i.e. ions were trapped in the HDC cell prior to transfer to the C-trap); C-trap entrance lens, $2 \mathrm{~V}$; C-trap exit lens, $20 \mathrm{~V}$. Note that the C-trap entrance and exit lenses as well as the C-trap bias varied with SID collision energy.

\section{Results \& Discussion}

\section{Device Design}

Gen 3 takes a minimalistic approach not only to significantly shorten the SID device but also to simplify SID tuning and improve the collection efficiency of protein complexes after surface collision. The last row in Figure 1 showcases the three new SID designs tested in this work. The SID cell in each case is a doubly-split lens, i.e. a single lens that has been split into 3 components: a deflector and an extractor on one side and a stainless steel surface on the other side. In the case of the Q-IM-TOF platform, the dynamic range enhancement lens after the quadrupole and before the Trap SRIG was reconfigured as shown in Figure 1, Gen 3. This device does not necessitate truncation of the Trap as has been required with Gen 1 and Gen 2 designs. On the FT-ICR, the front lens of the collision cell was split into the three requisite electrodes. That is, SID is accomplished in the front lens of the collision cell on this platform, and so the collision cell need not be truncated. On the Orbitrap, the transfer multipole prior to the Ctrap is replaced with a truncated hexapole of the same inscribed diameter (discussed later) with a split lens SID device placed just prior to the C-trap.

\section{Gen 3 SID in an FT-ICR}

Figures 1 and S1a, $\mathbf{b}$ show the simplicity of Gen 3 on the FT-ICR in comparison to earlier devices; it consists of only three electrodes - surface, deflector, and extractor - and a single nonconductive (PEEK or ceramic) spacer. This thin device replaces the original front endcap of the Bruker collision cell (Figure S1c), while the remaining portion of the collision cell as well as the rest of the instrument (Figure S2) remains untouched. The entire SID device is $3.25 \mathrm{~mm}$ in length (matching the thickness of the original endcap electrode), representing an order of magnitude reduction in size compared to Gen 1 and a $5 x$ decrease in size compared to Gen 2 (Figure S1a). 
Based on the simulations performed prior to device testing (described in the Supplemental Information, Figure S3, and Table S1), we hypothesized that the Gen 3 device would be more sensitive in SID mode compared to the Gen 2 device on the FT-ICR. The SID signal intensity was profiled using four different protein complexes: $53 \mathrm{kDa}$ charge-reduced streptavidin tetramer, $115 \mathrm{kDa}$ charge-reduced Creactive protein pentamer, $230 \mathrm{kDa}$ pyruvate kinase tetramer, and $330 \mathrm{kDa}$ glutamate dehydrogenase hexamer. The same nanospray tip was used to record full MS and SID intensities as a function of accumulation time for both Gen 2 and Gen 3 . The quadrupole was purposely placed in rf-only mode (placing $m / z 1,000$ at $q=0.706$ ) for these experiments, as the quadrupole can stretch the ion beam in the radial dimension with application of a resolving DC voltage.

A comparison of SID signal intensity across the usable energy range was first accomplished using streptavidin in EDDA (mainly $12+$ and $13+$ charge state precursors). Figure 2a compares the signal intensity observed in flythrough mode as well as SID at $25 \mathrm{~V}, 40 \mathrm{~V}, 80 \mathrm{~V}$, and $150 \mathrm{~V}$ (approximately 300$1950 \mathrm{eV}$ ). In all cases the Gen 3 signal is significantly higher. Gen 3 especially outperforms Gen 2 at the lower energies, $25 \mathrm{~V}$ and $40 \mathrm{~V}$, where Gen 1 and Gen 2 devices have historically struggled based on our experience and which will be evident on the other platforms discussed later. The capture efficiency after collision is clearly higher with the new design, as was indicated by the SIMION simulations. The spectra obtained at $40 \mathrm{~V}$ acceleration potential are overlaid in Figure $\mathbf{2 b}$. The Gen 3 spectrum in purple is noticeably higher in signal-to-noise.

For the comparisons of larger protein complexes the following acceleration voltages were used for illustration: C-reactive protein, $85 \mathrm{~V}$; pyruvate kinase and glutamate dehydrogenase, $135 \mathrm{~V}$. Figure $\mathbf{S 4}$ shows a comparison of absolute signal intensities for surface-induced dissociation of (a) C-reactive protein pentamer, (b) pyruvate kinase tetramer, and (c) glutamate dehydrogenase hexamer on both devices (Gen 2, green; Gen 3, purple) as a function of accumulation time. In all cases, Gen 3 achieves higher signal intensity at each accumulation time. Panels $(d, e, f)$ show spectra that correspond to the accumulation times highlighted by the black boxes. For C-reactive protein, approximately double the ion intensity was observed at $0.3 \mathrm{~s}$ accumulation time. For glutamate dehydrogenase and pyruvate kinase, the intensity was nearly $5 x$ higher for Gen 3 in both cases (at $1 \mathrm{~s}$ and $2 \mathrm{~s}$ accumulation times, respectively), which is evident from the spectral comparisons in panels (e) and (f).

\section{Surface-induced dissociation coupled to ultrahigh (FT-ICR) resolution}

The 15 T FT-ICR provides resolution far exceeding other mass analyzers. We chose several cyclic protein complexes to demonstrate the utility of ultrahigh resolution (and, later, ion mobility on the QIM-TOF), as these tend to dissociate by SID into all possible oligomeric states and so overlap in $\mathrm{m} / \mathrm{z}$ due to symmetric charge partitioning.

HFQ65 is a cyclic $43 \mathrm{kDa}$ homohexameric RNA chaperone that is a truncated form of HFQ102. ${ }^{46,47}$ The full scan of the precursor (charge reduced using TEAA) in Figure 3a shows $8+$ through $12+$ hexamers as prominent native species. Because the complex is cyclic, the SID spectrum of the isolated $10+$ hexamer using a $35 \mathrm{~V}$ potential difference yields monomer through pentamer, and many peaks consist of multiple oligomeric states which require either ion mobility or isotopic resolution to deconvolute. The peak at $m / z 3594$, for example, clearly consists of monomer $2+$, dimer $4+$, and trimer $6+$, all of which are resolved with a 9 s transient. 
HFQ102 (66 kDa) is similarly a homohexameric protein complex but has molecular 'tails' on each subunit, which are not present on HFQ65. Figure $\mathbf{3 b}$ shows the full scan and SID spectrum of the chargereduced complex, with hexamer $10+$ through $13+$ observed as precursors. The peak $\mathrm{m} / \mathrm{z} 5518$ consists of several species, monomer $2+$, dimer $4+$, trimer $6+$, and tetramer $8+$. While monomer through trimer are baseline resolved from each other, there is overlap once a fourth oligomer is added to the mix, particularly as the FT-ICR's resolution decreases at higher $\mathrm{m} / \mathrm{z}$. Nevertheless, these examples highlight the utility of ultrahigh resolution for analyzing SID products of protein complexes.

Trp RNA binding attenuation protein (TRAP) is a $91 \mathrm{kDa}$ homo-11mer that binds tryptophan and participates in allosteric gene regulation. TRAP is another cyclic protein complex whose structure is conserved in the gas phase. ${ }^{48,49}$ The mass spectrum of the holo trpTRAP protein complex with 14 equivalents of trp (i.e. trp in excess) in 200 mM EDDA in Figure S5a indicates primary charge states of $16+$ through 20+. As expected, the collision-induced dissociation spectrum of the 18+ charge state in Figure $\mathbf{S 5 b}$ shows asymmetrically charged monomers and decamers. On average, the monomers take approximately $1 / 3$ of the charge despite making up only $1 / 11$ of the mass of the total complex. Despite the many possible cleavages of the protein, no oligomers beyond decamer and monomer were observed, highlighting the limited utility of CID in this particular case. Surface-induced dissociation of the 18+ charge state (Figure S5c), on the other hand, gives a wide variety of symmetrically charged products ranging from monomer through decamer, though most of the ion intensity is monomer through heptamer. The observation of every oligomeric fragment is consistent with a cyclic 11 mer. The insets in panels ( $d-f)$ show expanded views of various peaks obtained at higher resolution ( 9 s transient).

Implementation of a split lens SID design on a Q-IM-TOF platform

Because the split lens SID design is minimalistic and small ( $3 \mathrm{~mm}$ length), it is easier to install in various MS platforms compared to prior device designs from our laboratory. We reconfigured the dynamic range enhancement (DRE) lens in Waters Synapt G1 and G2 Q-IM-TOF mass spectrometers to perform SID experiments (Figure 1, bottom left). An instrument schematic of the Synapt G2 is given in Figure S6a. The DRE lens is located behind the quadrupole mass filter and in front of the trap SRIG; it is composed of a split lens followed by a second lens (Figure S6b, top). The DRE lens reconfigured for SID consists of two of the original lens elements which previously comprised a split lens but are now used as deflector and extractor for SID (Figure 4a and Figure S6b, bottom). Only a single stainless-steel electrode was fabricated and inserted as the surface opposite the deflector and extractor. The replacement of the DRE lens did not affect flythrough signal for C-reactive protein (Figure S7) over three replicate nESI tips.

To reduce nanospray variability on the Synapt G2, both Gen 1 and Gen 3 SID devices were kept in the instrument simultaneously. A comparison was made between SID spectra and signal intensities for Gen 1 and Gen 3 SID devices by using 53 kDa streptavidin tetramer in EDDA (average charge state $13+)$ and IM-TOF mode across a wide range of energies. The spectra were again obtained without quadrupole isolation to remove any quadrupole effects from the comparison. The measured Gen 3 signal intensities were significantly higher at the lower energies (SID $25 \mathrm{~V}$ and $40 \mathrm{~V}$ comparison are shown in Figure $\mathbf{8 8 a , b}$ ) and the highest energy (150 V) - in agreement with our observations on the FTICR - but the spectra obtained at $80 \mathrm{~V}$ were comparable. This comparison is summarized in Figure S8c.

Signal improvement was also noticeable with larger protein complexes. SID of both pyruvate kinase and glutamate dehydrogenase yielded higher absolute signal than with the Gen 1 device. For 
pyruvate kinase (average charge state $29+$ ) the SID signal at $135 \mathrm{~V}$ increased by $\sim 30 \%$, and at $200 \mathrm{~V}$ the signal increased by $20 \%$ (Figure S9a). For SID of GDH (average charge state $40+$ ) at $135 \mathrm{~V}$ the signal increased by $\sim 50 \%$ (Figure $\mathbf{S 9 b}$ ).

On the FT-ICR the ultrahigh resolution could be used to determine oligomeric states of subcomplexes generated after SID through isotopic spacings; on the $\mathrm{G} 2$, traveling wave ion mobility is used to a similar effect. Figure $4 \mathbf{b}$ shows the SID spectrum of charge reduced HFQ65 in IM-TOF mode with quadrupole selection of the 10+ charge state. Clear separation is observed in the ion mobility spectrum in Figure 4c, and there is good agreement between FT-ICR and Q-IM-TOF spectra.

This experiment was repeated with the 11mer holoTRAP complex (with 14 eq trp in $200 \mathrm{mM}$ EDDA) also investigated on the FT-ICR, and the results are shown in Figure 5 with the isolated 18+ charge state. On the G2 all oligomeric fragments, from monomer through decamer, were observed after SID and are more clearly separated in ion mobility space than with the ultrahigh resolution of the FT-ICR. Clearly, the replacement of the DRE lens does not affect the ion mobility capabilities of the instrument.

Gen 3 was also installed on a Synapt G1 to demonstrate the wide applicability of the current SID design on multiple Synapt platforms. apoTRAP 11mer was analyzed on the G1 in SID-IM-TOF mode (Figure S10). As before, due to the cyclic structure of the complex, many types of oligomer fragments are observed after surface collision of the charge reduced precursors. Note that no quadrupole selection was used in this case. These data are in good agreement with SID data from both the FT-ICR and the G2.

\section{Implementation of a split lens SID design on an Orbitrap platform}

A Gen 3 SID device was also installed into an Exactive EMR Orbitrap platform, previously modified to include a selection quadrupole. The first generation design (Figure 6, 'Gen 1') incorporated 12 dc-only electrodes ${ }^{26,42}$ arranged in a manner similar to the Gen 1 design on the FT-ICR and Synapts. The front and rear 3 elements ( 6 total) comprise Einzel lenses with the first and third electrodes of each trio electrically connected. Hence, the SID cell uses 10 independent voltages to power the 12 electrodes plus one more externally supplied voltage to vary the C-trap offset. The Gen 1 SID device replaced the transfer multipole prior to the C-trap (red star in the instrument diagram Figure S11a). While the device is robust and capable of SID across a large energy and mass range, reduction in the number of electrodes for SID would be beneficial for ease-of-use. We incorporated a Gen 3 SID design in the same location as the Gen 1 SID cell by designing and fabricating a truncated hexapole (rod diameter $4.75 \mathrm{~mm}$, inscribed diameter $8.75 \mathrm{~mm}$ ) to make room for the doubly-split lens SID device just prior to the C-trap (model shown in Figure 6, 'Gen 3'; fabricated device shown in Figure S11b). Figure S11b also shows the original octupole that the device replaces.

In transmission mode, very little difference in overall signal intensity and relative abundances was observed with a CsPFHA cluster calibration solution when comparing Gen 1 and Gen 3 on the EMR (Figure S12). Transmission of large protein complexes was similarly unaffected. Figure S13 shows transmission of 330 kDa glutamate dehydrogenase hexamer with Gen 1 and Gen 3 devices installed. Over three replicate tips, no measurable difference was observed.

The SID performance of Gen 1 and Gen 3 were compared with charge-reduced streptavidin (60 $\mathrm{mM}$ EDDA as reducing agent) without ion isolation to prevent quadrupole effects from convoluting the 
results. Although both devices return similar intensities in flythrough and SID 85 V spectra, Gen 3 is particularly notable for its increased performance at the lower energy (SID $45 \mathrm{~V}$ ), an observation now made across three separate instrument platforms. An almost 4-fold increase in sensitivity was observed, (Figure S14).

SID spectra of the 13+ homopentamer of cholera toxin B were acquired on the EMR (Figure S15). The spectra are similar to those obtained on the FT-ICR (Figure S16) in that all oligomeric fragments are produced due to the cyclic arrangement of the subunits. While the FT-ICR can resolve

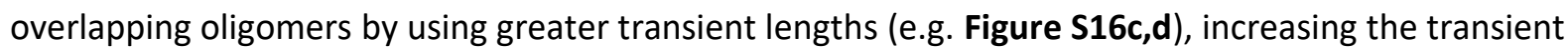
length on the Orbitrap to the $140 \mathrm{k}$ setting (512 ms transient, Figure S15c) results in substantial bias toward the lower mass fragments due to the high path length in the Orbitrap cell. ${ }^{42,50}$

Surface-induced dissociation of the 9+ charge state of HFQ65 on the EMR is given in Figure $\mathbf{6 b , c}$ with the full MS shown in Figure 6a. While the low-resolution Orbitrap spectrum in panel (b) agrees well with the spectrum obtained on the FT-ICR (Figure S17a), at higher resolution spectral biases are observed. Insets in panels (b) and (c) show the resolution achieved for $\mathrm{m} / \mathrm{z} 3594$ at the different transient lengths. Dimer is only resolved in the higher resolution spectrum, and trimer is not resolved in either spectrum. Comparable SID spectra of the isolated 10+ charge state (the most abundant) on the FT-ICR required a $2.2 \mathrm{~s}$ and $9 \mathrm{~s}$ transient (Figure S17a,b).

The SID spectrum of 11mer holoTRAP (Figure S18) was also similar to the spectra obtained on the FT-ICR and Synapt, with monomer through heptamer being the most prominent fragments, though note that all oligomeric fragments were again observed due to the cyclic structure of the protein complex.

Gen 3 on the EMR works with large complexes at high acceleration potentials as well. Figure S19 shows the full scan and SID spectra of the 38+ charge state of $330 \mathrm{kDa}$ glutamate dehydrogenase at $5130 \mathrm{eV}$ and $6650 \mathrm{eV}$. In both low and high energy cases, the predominant fragments are trimers, in agreement with the dimer of trimers arrangement of the subunits. ${ }^{42,45,51} \mathrm{Gen} 3$ on the EMR is therefore capable of transmitting and fragmenting ions over a wide range of masses and energies yet demonstrates a simpler SID design to increase usability and improve SID automation for data-dependent MS/MS analyses in the future.

\section{Conclusion}

A miniaturized split lens surface-induced dissociation device has been fabricated and experimentally compared to a previous SID design on an FT-ICR, Synapt G1 and G2 Q-IM-TOF mass spectrometers, and a modified (Q) Exactive EMR Orbitrap. In addition to a reduction in length to $3 \mathrm{~mm}$, the device exhibits up to $5 x$ improvement in SID intensities compared to the second-generation device on the FT-ICR operated at the same accumulation time and improves low energy performance compared to Gen 1 devices across all platforms. Ultrahigh resolution coupled with surface-induced dissociation of protein complexes allows for the deconvolution of overlapping oligomers and can be particularly valuable in conjunction with corroborating results from ion mobility, as demonstrated here with TRAP 11mer and HFQ65. The SID design can be generalized to other multipole ion guides, collision cells, and stacked ring ion guides that have an entrance or exit lens large enough to accommodate the 
split lens electrodes and can more easily be implemented in other instrument platforms due to its reduced footprint. The reduction in the number of independent electrodes also simplifies tuning and paves the way for easier SID automation.

\section{Acknowledgements}

The authors thank the OSU Arts and Sciences College Machine Shop for fabricating the parts described in this manuscript. The authors also acknowledge helpful discussions with Bruker Corporation (Mel Park and Mark Ridgway in particular), Thermo Fisher Scientific and Waters Corporation. We thank Jorjethe Roca (Johns Hopkins University) and Sarah Woodson (Johns Hopkins University) for the Hfq samples (NIH R01 GM120425), and Melody Holmquist, Mark Foster (OSU), and Paul Gollnick (SUNY Buffalo) for the TRAP sample (NIH R01 GM120923). The FT-ICR was purchased using funds from NIH Award S10 OD018507. This work was supported by the NIH P41 Resource for Native Mass Spectrometry Guided Structural Biology, P41GM128577.

\section{References}

(1) Mabud, MD. A.; Dekrey, M. J.; Cooks, R. G. Surface-Induced Dissociation of Molecular lons. Int J Mass Spectrom Ion Process. 1985, 67, 285-294.

(2) Cooks, R. G.; Terwilliger, D. T.; Ast, T.; Beynon, J. H.; Keough, T. Surface Modified MassSpectrometry. J. Am. Chem. Soc. 1975, 97 (6), 1583-1585. https://doi.org/10.1021/ja00839a056.

(3) DeKrey, M. J.; Kenttämaa, H. I.; Wysocki, V. H.; Cooks, R. G. Energy Deposition in [Fe(CO)5]+' upon Collision with a Metal Surface. Org. Mass Spectrom. 1986, 21 (4), 193-195. https://doi.org/10.1002/oms.1210210405.

(4) Wysocki, V. H.; Jones, C. M.; Galhena, A. S.; Blackwell, A. E. Surface-Induced Dissociation Shows Potential to Be More Informative than Collision-Induced Dissociation for Structural Studies of Large Systems. J Am Soc Mass Spectrom 2008, 19 (7), 903-913. https://doi.org/10.1016/j.jasms.2008.04.026.

(5) Laskin, J.; Futrell, J. H. Surface-Induced Dissociation of Peptide lons: Kinetics and Dynamics. J. Am. Soc. Mass Spectrom. 2003, 14 (12), 1340-1347.

(6) Laskin, J.; Denisov, E.; Futrell, J. A Comparative Study of Collision-Induced and Surface-Induced Dissociation. 1. Fragmentation of Protonated Dialanine. J. Am. Chem. Soc. 2000, 122 (40), $9703-$ 9714. https://doi.org/10.1021/ja001384w.

(7) McCormack, A. L.; Jones, J. L.; Wysocki, V. H. Surface-Induced Dissociation of Multiply Protonated Peptides. J. Am. Soc. Mass Spectrom. 1992, 3 (8), 859-862.

(8) Wysocki, V. H.; Joyce, K. E.; Jones, C. M.; Beardsley, R. L. Surface-Induced Dissociation of Small Molecules, Peptides, and Non-Covalent Protein Complexes. J Am Soc Mass Spectrom 2008, 19 (2), 190-208. https://doi.org/10.1016/j.jasms.2007.11.005.

(9) Rožman, M.; Gaskell, S. J. Charge State Dependent Top-down Characterisation Using Electron Transfer Dissociation. Rapid Commun. Mass Spectrom. 2012, 26 (3), 282-286. https://doi.org/10.1002/rcm.5330.

(10) Breuker, K.; McLafferty, F. W. Native Electron Capture Dissociation for the Structural Characterization of Noncovalent Interactions in Native Cytochrome c. Angew. Chem. Int. Ed. 2003, 42 (40), 4900-4904. https://doi.org/10.1002/anie.200351705. 
(11) Li, H.; Sheng, Y.; McGee, W.; Cammarata, M.; Holden, D.; Loo, J. A. Structural Characterization of Native Proteins and Protein Complexes by Electron Ionization Dissociation-Mass Spectrometry. Anal. Chem. 2017, 89 (5), 2731-2738. https://doi.org/10.1021/acs.analchem.6b02377.

(12) Lermyte, F.; Williams, J. P.; Brown, J. M.; Martin, E. M.; Sobott, F. Extensive Charge Reduction and Dissociation of Intact Protein Complexes Following Electron Transfer on a Quadrupole-Ion Mobility-Time-of-Flight MS. J. Am. Soc. Mass Spectrom. 2015, 26 (7), 1068-1076. https://doi.org/10.1007/s13361-015-1124-z.

(13) Lermyte, F.; Sobott, F. Electron Transfer Dissociation Provides Higher-Order Structural Information of Native and Partially Unfolded Protein Complexes. PROTEOMICS 2015, 15 (16), 2813-2822. https://doi.org/10.1002/pmic.201400516.

(14) Sobott, F.; Robinson, C. V. Characterising Electrosprayed Biomolecules Using Tandem-MS-the Noncovalent GroEL Chaperonin Assembly. Int J Mass Spectrom 2004, 236 (1-3), 25-32. https://doi.org/10.1016/j.ijms.2004.05.010.

(15) Light-Wahl, K. J.; Schwartz, B. L.; Smith, R. D. Observation of the Noncovalent Quaternary Associations of Proteins by Electrospray Ionization Mass Spectrometry. J. Am. Chem. Soc. 1994, 116 (12), 5271-5278. https://doi.org/10.1021/ja00091a035.

(16) Versluis, C.; Heck, A. J. R. Gas-Phase Dissociation of Hemoglobin11Dedicated to Nico Nibbering with Many Thanks for His Support and Infectious Enthusiasm over the Years. Int. J. Mass Spectrom. 2001, 210-211, 637-649. https://doi.org/10.1016/S1387-3806(01)00428-6.

(17) Brodbelt, J. S. Ion Activation Methods for Peptides and Proteins. Anal Chem 2016, 88 (1), 30-51. https://doi.org/10.1021/acs.analchem.5b04563.

(18) Sipe, S. N.; Brodbelt, J. S. Impact of Charge State on $193 \mathrm{Nm}$ Ultraviolet Photodissociation of Protein Complexes. Phys. Chem. Chem. Phys. 2019, 21 (18), 9265-9276. https://doi.org/10.1039/C9CP01144G.

(19) Li, H.; Nguyen, H. H.; Ogorzalek Loo, R. R.; Campuzano, I. D. G.; Loo, J. A. An Integrated Native Mass Spectrometry and Top-down Proteomics Method That Connects Sequence to Structure and Function of Macromolecular Complexes. Nat Chem 2018, 10 (2), 139-148. https://doi.org/10.1038/nchem.2908.

(20) Quintyn, R. S.; Zhou, M.; Yan, J.; Wysocki, V. H. Surface-Induced Dissociation Mass Spectra as a Tool for Distinguishing Different Structural Forms of Gas-Phase Multimeric Protein Complexes. Anal Chem 2015, 87 (23), 11879-11886.

(21) Stiving, A. Q.; VanAernum, Z. L.; Busch, F.; Harvey, S. R.; Sarni, S. H.; Wysocki, V. H. SurfaceInduced Dissociation: An Effective Method for Characterization of Protein Quaternary Structure. Anal Chem 2019, 91 (1), 190-209. https://doi.org/10.1021/acs.analchem.8b05071.

(22) Jones, C. M.; Beardsley, R. L.; Galhena, A. S.; Dagan, S.; Cheng, G.; Wysocki, V. H. Symmetrical Gas-Phase Dissociation of Noncovalent Protein Complexes via Surface Collisions. J Am Chem Soc 2006, 128 (47), 15044-15045. https://doi.org/10.1021/ja064586m.

(23) Song, Y.; Nelp, M. T.; Bandarian, V.; Wysocki, V. H. Refining the Structural Model of a Heterohexameric Protein Complex: Surface Induced Dissociation and Ion Mobility Provide Key Connectivity and Topology Information. ACS Cent Sci 2015, 1 (9), 477-487. https://doi.org/10.1021/acscentsci.5b00251.

(24) Sahasrabuddhe, A.; Hsia, Y.; Busch, F.; Sheffler, W.; King, N. P.; Baker, D.; Wysocki, V. H. Confirmation of Intersubunit Connectivity and Topology of Designed Protein Complexes by Native MS. Proc Natl Acad Sci USA 2018, 115 (6), 1268-1273.

(25) Harvey, S. R.; Seffernick, J. T.; Quintyn, R. S.; Song, Y.; Ju, Y.; Yan, J.; Sahasrabuddhe, A. N.; Norris, A.; Zhou, M.; Behrman, E. J.; Lindert, S.; Wysocki, V. H. Relative Interfacial Cleavage Energetics of Protein Complexes Revealed by Surface Collisions. Proc Natl Acad Sci USA 2019, 116 (17), 81438148. https://doi.org/10.1073/pnas.1817632116. 
(26) Busch, F.; VanAernum, Z. L.; Ju, Y.; Yan, J.; Gilbert, J. D.; Quintyn, R. S.; Bern, M.; Wysocki, V. H. Localization of Protein Complex Bound Ligands by Surface-Induced Dissociation High-Resolution Mass Spectrometry. Anal Chem 2018, 90 (21), 12796-12801.

https://doi.org/10.1021/acs.analchem.8b03263.

(27) Galhena, A. S.; Dagan, S.; Jones, C. M.; Beardsley, R. L.; Wysocki, V. H. Surface-Induced Dissociation of Peptides and Protein Complexes in a Quadrupole/Time-of-Flight Mass Spectrometer. Anal Chem 2008, 80 (5), 1425-1436. https://doi.org/10.1021/ac701782q.

(28) Hernández, H.; Robinson, C. V. Determining the Stoichiometry and Interactions of Macromolecular Assemblies from Mass Spectrometry. Nat. Protoc. 2007, 2 (3), 715.

(29) Benesch, J. L.; Aquilina, J. A.; Ruotolo, B. T.; Sobott, F.; Robinson, C. V. Tandem Mass Spectrometry Reveals the Quaternary Organization of Macromolecular Assemblies. Chem Biol 2006, 13 (6), 597-605.

(30) Rostom, A. A.; Robinson, C. V. Detection of the Intact GroEL Chaperonin Assembly by Mass Spectrometry. J Am Chem Soc 1999, 121 (19), 4718-4719. https://doi.org/10.1021/ja990238r.

(31) Fort, K. L.; Van de Waterbeemd, M.; Boll, D.; Reinhardt-Szyba, M.; Belov, M. E.; Sasaki, E.; Zschoche, R.; Hilvert, D.; Makarov, A. A.; Heck, A. J. Expanding the Structural Analysis Capabilities on an Orbitrap-Based Mass Spectrometer for Large Macromolecular Complexes. Analyst 2018, 143 (1), 100-105.

(32) Wysocki, V. H.; Ding, J.-M.; Jones, J. L.; Callahan, J. H.; King, F. L. Surface-Induced Dissociation in Tandem Quadrupole Mass Spectrometers: A Comparison of Three Designs. J Am Soc Mass Spectrom 1992, 3 (1), 27-32.

(33) Bier, M.; Amy, J.; Cooks, R.; Syka, J.; Ceja, P.; Stafford, G. A Tandem Quadrupole Mass Spectrometer for the Study of Surface-Induced Dissociation. Int J Mass Spectrom Ion Process 1987, 77 (1), 31-47.

(34) Mohammed, S.; Chalmers, M. J.; Gielbert, J.; Ferro, M.; Gora, L.; Smith, D. C.; Gaskell, S. J. A Novel Tandem Quadrupole Mass Spectrometer Allowing Gaseous Collisional Activation and Surface Induced Dissociation. J. Mass Spectrom. 2001, 36 (12), 1260-1268.

(35) Lammert, S. A.; Cooks, R. G. Surface-Induced Dissociation of Molecular lons in a Quadrupole Ion Trap Mass Spectrometer. J Am Soc Mass Spectrom 1991, 2 (6), 487-491. https://doi.org/10.1016/1044-0305(91)80036-7.

(36) Hilger, R. T.; Santini, R. E.; McLuckey, S. A. Tandem Mass Spectrometry in an Electrostatic Linear Ion Trap Modified for Surface-Induced Dissociation. Anal. Chem. 2014, 86 (17), 8822-8828.

(37) Williams, E. R.; Fang, L.; Zare, R. N. Surface Induced Dissociation for Tandem Time-of-Flight Mass Spectrometry. Int. J. Mass Spectrom. Ion Process. 1993, 123 (3), 233-241.

(38) Chorush, R. A.; Little, D. P.; Beu, S. C.; Wood, T. D.; McLafferty, F. W. Surface-Induced Dissociation of Multiply-Protonated Proteins. Anal Chem 1995, 67 (6), 1042-1046.

(39) Laskin, J.; Denisov, E. V.; Shukla, A. K.; Barlow, S. E.; Futrell, J. H. Surface-Induced Dissociation in a Fourier Transform Ion Cyclotron Resonance Mass Spectrometer: Instrument Design and Evaluation. Anal Chem 2002, 74 (14), 3255-3261.

(40) Williams, E. R.; Henry, K. D.; McLafferty, F. W.; Shabanowitz, J.; Hunt, D. F. Surface-Induced Dissociation of Peptide Ions in Fourier-Transform Mass Spectrometry. J Am Soc Mass Spectrom 1990, 1 (5), 413-416. https://doi.org/10.1016/1044-0305(90)85022-E.

(41) Ijames, C. F.; Wilkins, C. L. Surface-Induced Dissociation by Fourier Transform Mass Spectrometry. Anal. Chem. 1990, 62 (13), 1295-1299.

(42) VanAernum, Z. L.; Gilbert, J. D.; Belov, M. E.; Makarov, A. A.; Horning, S. R.; Wysocki, V. H. Surface-Induced Dissociation of Noncovalent Protein Complexes in an Extended Mass Range Orbitrap Mass Spectrometer. Anal Chem 2019, 91 (5), 3611-3618. https://doi.org/10.1021/acs.analchem.8b05605. 
(43) Yan, J.; Zhou, M.; Gilbert, J. D.; Wolff, J. J.; Somogyi, A.; Pedder, R. E.; Quintyn, R. S.; Morrison, L. J.; Easterling, M. L.; Pasa-Tolic, L.; Wysocki, V. H. Surface-Induced Dissociation of Protein Complexes in a Hybrid Fourier Transform Ion Cyclotron Resonance Mass Spectrometer. Anal Chem 2017, 89 (1), 895-901. https://doi.org/10.1021/acs.analchem.6b03986.

(44) Stiving, A. Q.; Gilbert, J. D.; Wysocki, V. H. Development of Ion Carpet-Surface Induced Dissociation (SID) Devices For Simplified Tuning; 2018.

(45) Snyder, D. T.; Panczyk, E.; Stiving, A. Q.; Gilbert, J. D.; Somogyi, A.; Kaplan, D.; Wysocki, V. Design and Performance of a Second-Generation Surface-Induced Dissociation Cell for Fourier Transform Ion Cyclotron Resonance Mass Spectrometry of Native Protein Complexes. Anal. Chem. 2019, 91 (21), 14049-14057. https://doi.org/10.1021/acs.analchem.9b03746.

(46) Sauer, E.; Weichenrieder, O. Structural Basis for RNA 3'-End Recognition by Hfq. Proc Natl Acad Sci USA 2011, 108 (32), 13065-13070. https://doi.org/10.1073/pnas.1103420108.

(47) Vogel, J.; Luisi, B. F. Hfq and Its Constellation of RNA. Nat Rev Microbiol 2011, 9, 578. https://doi.org/10.1038/nrmicro2615.

(48) Kleckner, I. R.; Gollnick, P.; Foster, M. P. Mechanisms of Allosteric Gene Regulation by NMR Quantification of Microsecond-Millisecond Protein Dynamics. J Mol Biol 2012, 415 (2), 372-381.

(49) Ruotolo, B. T.; Giles, K.; Campuzano, I.; Sandercock, A. M.; Bateman, R. H.; Robinson, C. V. Evidence for Macromolecular Protein Rings in the Absence of Bulk Water. Science 2005, 310 (5754), 1658-1661. https://doi.org/10.1126/science.1120177.

(50) Makarov, A.; Denisov, E. Dynamics of lons of Intact Proteins in the Orbitrap Mass Analyzer. J. Am. Soc. Mass Spectrom. 2009, 20 (8), 1486-1495.

(51) Ma, X.; Zhou, M.; Wysocki, V. H. Surface Induced Dissociation Yields Quaternary Substructure of Refractory Noncovalent Phosphorylase B and Glutamate Dehydrogenase Complexes. J Am Soc Mass Spectrom 2014, 25 (3), 368-379. https://doi.org/10.1007/s13361-013-0790-y. 


\section{Figures}

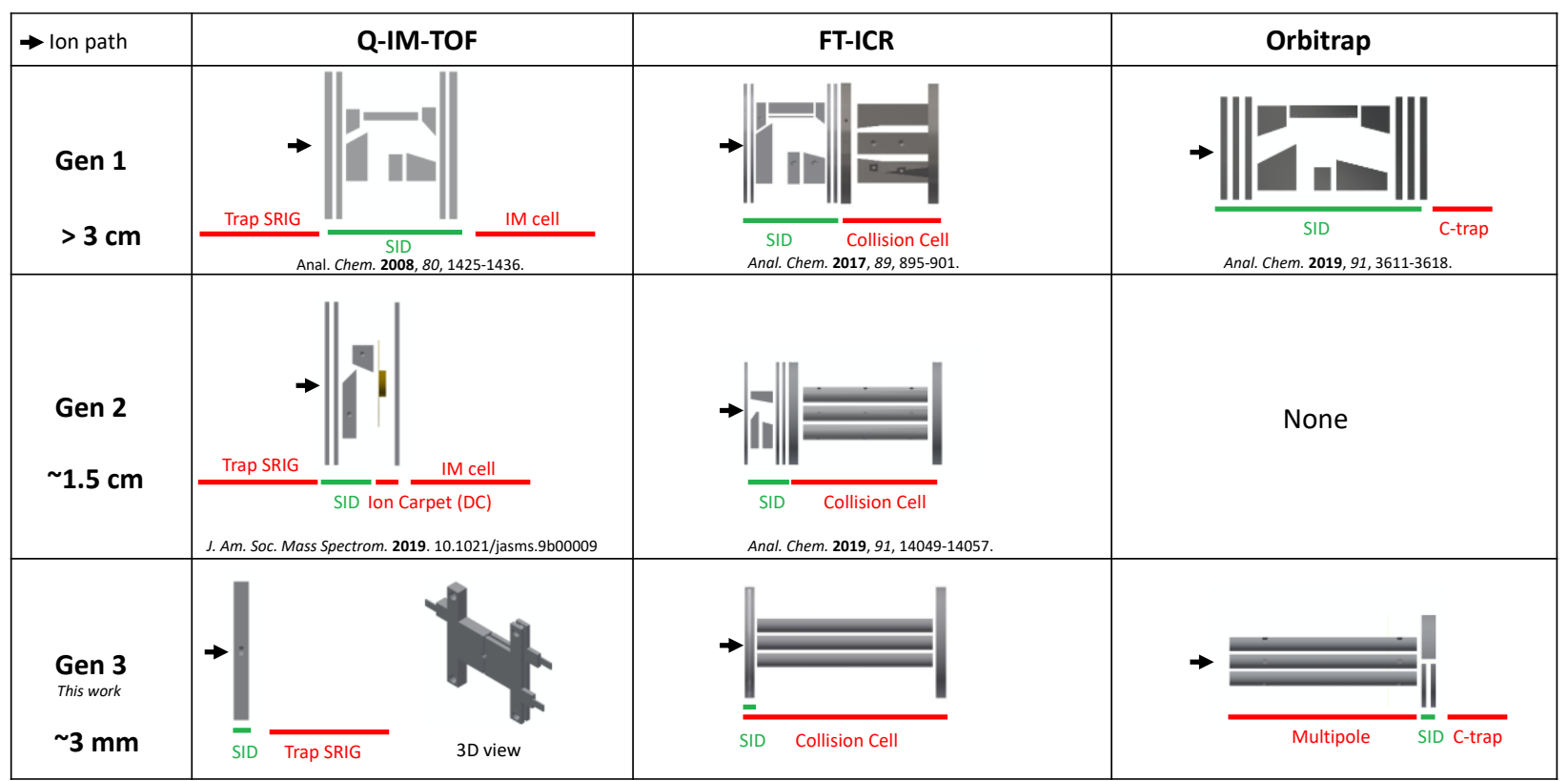

Figure 1: Three generations of SID designs developed in the Wysocki laboratory. 'Gen 1' devices have SID lengths $>3 \mathrm{~cm}$ and consist of 10-12 SID electrodes. 'Gen 2' devices consist of tilted surfaces with fewer electrodes and occupy approximately half the volume as their 'Gen 1' counterparts. 'Gen 3' devices, reported here, occupy approximately $3 \mathrm{~mm}$ along the ion path and consist of only three lenses, surface, deflector, and extractor. SRIG = stacked ring ion guide. SID = surface-induced dissociation cell. $\mathrm{IM}=$ ion mobility. 

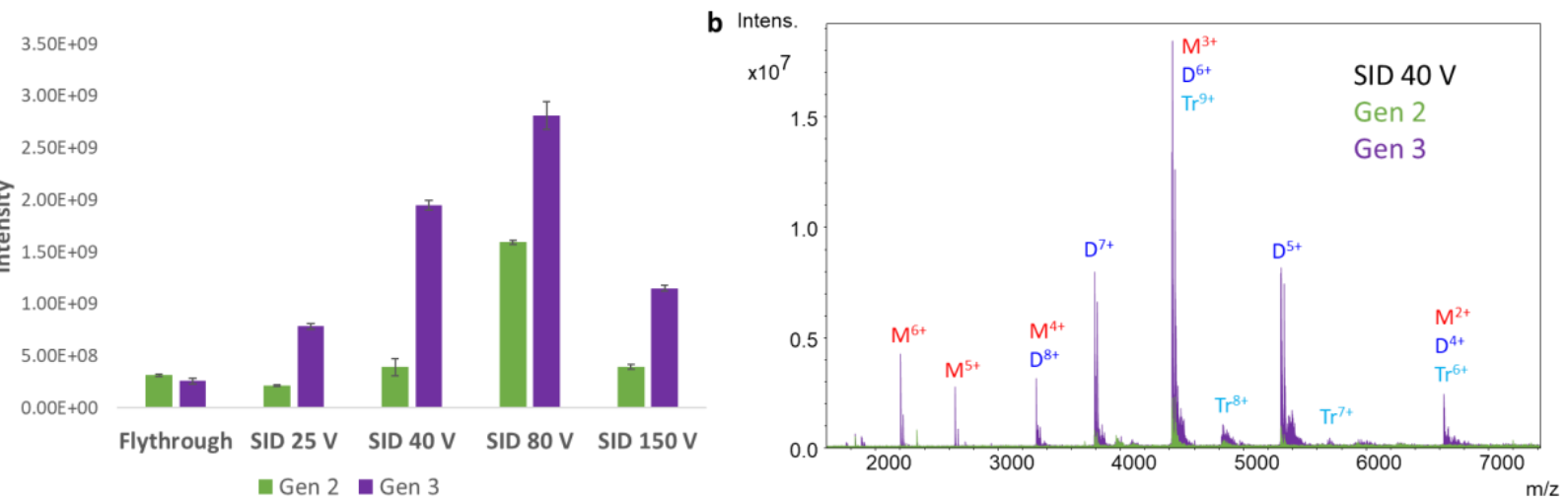

Figure 2: Comparison of sensitivity of Gen 2 and Gen 3 SID devices on the FT-ICR using streptavidin in EDDA (mainly 12+ and 13+ precursors, no isolation): (a) Comparison of flythrough and SID intensities under comparable conditions, and (b) comparison of representative SID spectra at $40 \mathrm{~V}$ acceleration ( $Q$ (tetramer) produces two dimers, $\mathrm{D}$ (e.g., $\mathrm{Q}^{12+} \rightarrow 2 \mathrm{D}^{6+}$ and $\mathrm{Q}^{13+} \rightarrow \mathrm{D}^{6+}$ plus $\mathrm{D}^{7+}$ ), and monomer $(\mathrm{M})$ plus trimer (T).) 
a
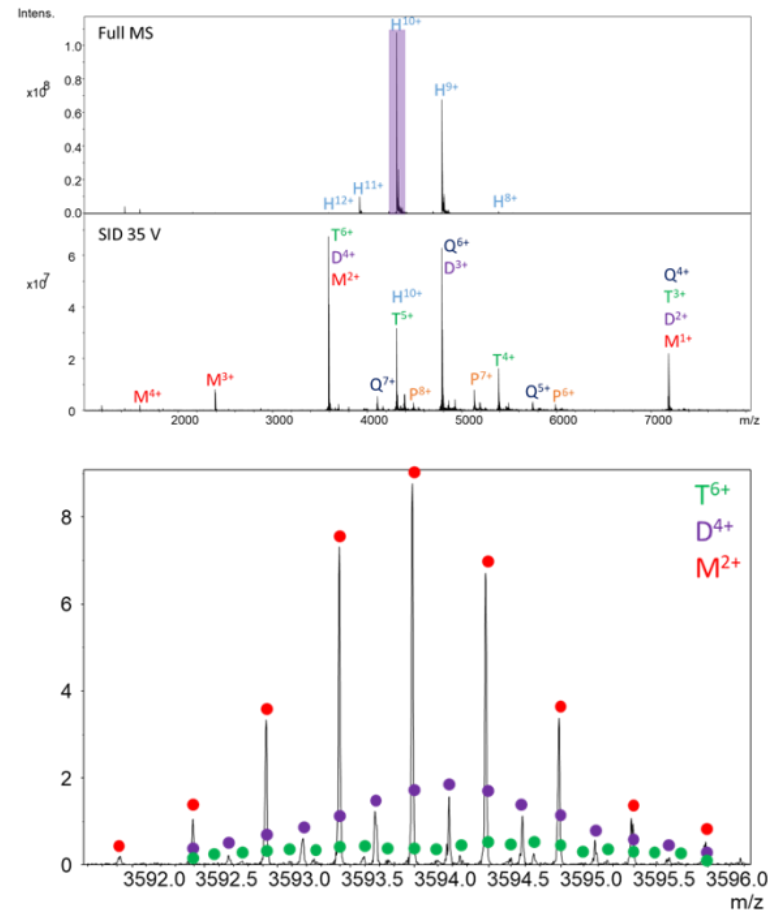

b
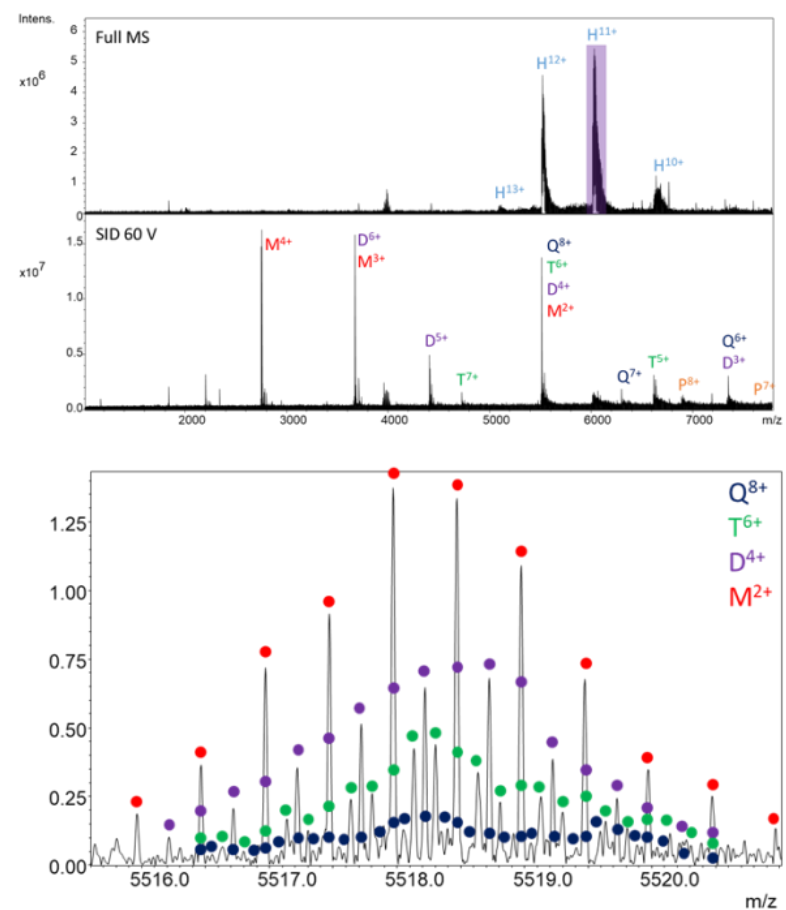

Figure 3: Full scans and surface-induced dissociation spectra of hexameric (a) HFQ65 and (b) HFQ102 using the Gen 3 device on the FT-ICR. The four bottom spectra are zoomed in to show the isotopic resolution of the $15 \mathrm{TICR}$ using a $9 \mathrm{~s}$ transient. The SID spectra are an average of 30 scans at $4 \mathrm{M}$ transient length (low $\mathrm{m} / \mathrm{z} 1,000$ ); the full scans are an average of 10 at $1 \mathrm{M}$ transient length. $\mathrm{M}=$ monomer, $\mathrm{D}=$ dimer, $\mathrm{T}=$ trimer, $\mathrm{Q}=$ tetramer, $\mathrm{P}=$ pentamer, $\mathrm{H}=$ hexamer 

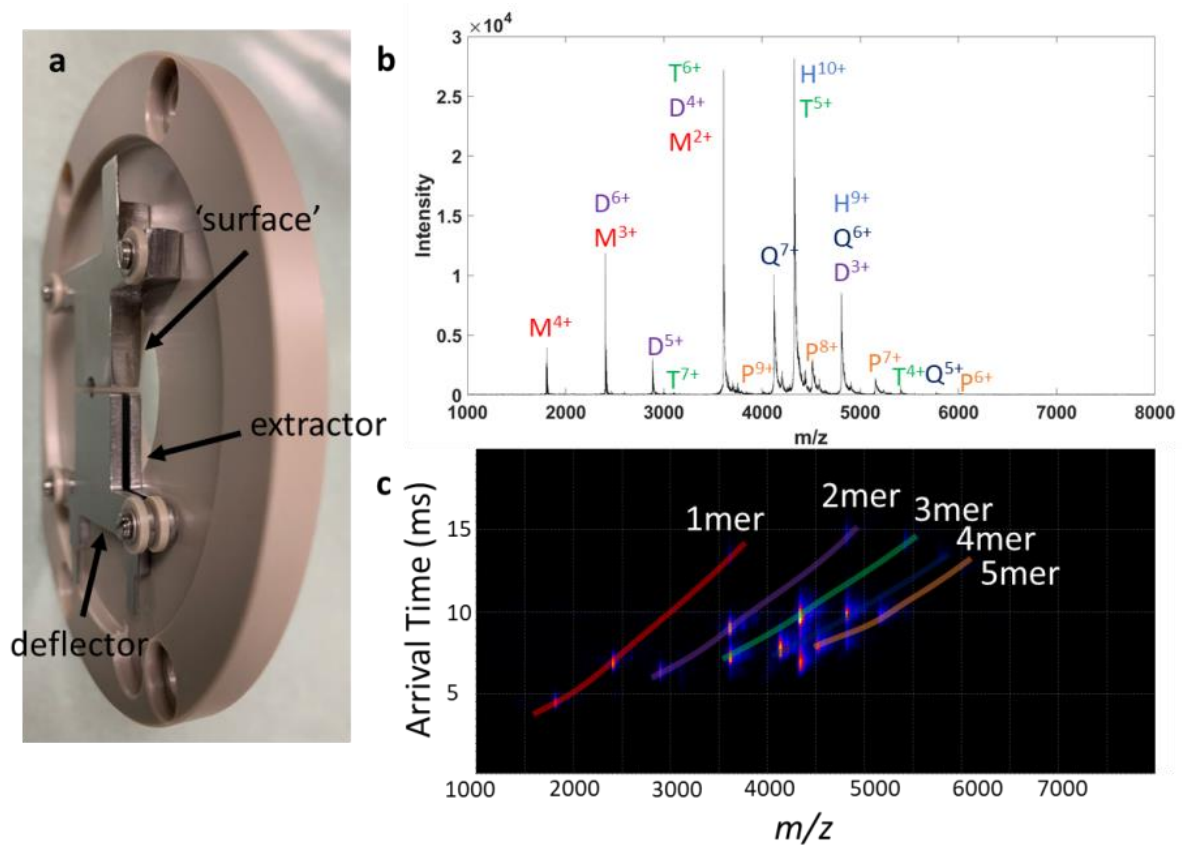

Figure 4: Gen 3 SID on a Synapt G2 platform. (a) photograph of DRE (dynamic range enhancement) lens reconfigured to perform SID, (b) SID spectrum of the 10+ charge state of HFQ65 (with TEAA as charge reducing reagent) in IM-TOF mode recorded on the $\mathrm{G} 2$, and (c) mobiligram associated with the SID spectrum. 


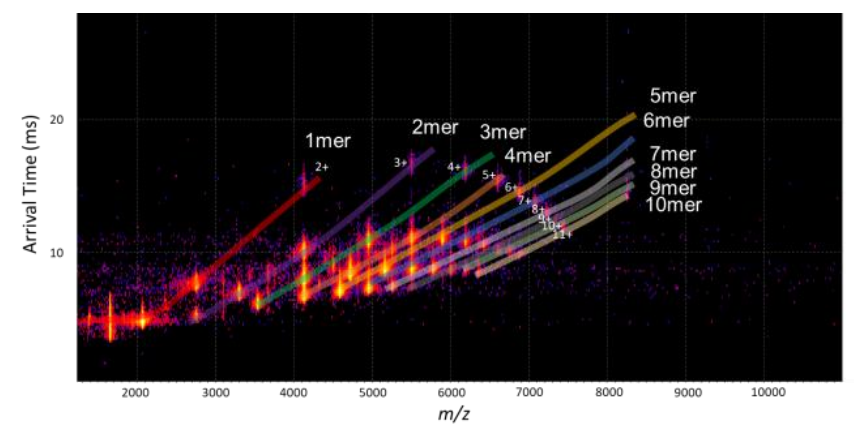

Figure 5: SID-IM-TOF of the 18+ charge state of holoTRAP 11mer (in 200 mM EDDA with 14 equivalents of $t r p$ ) using Gen 3 on the Synapt G2. All oligomeric fragments are observed in mobility space, consistent with the cyclic arrangement of the subunits. 


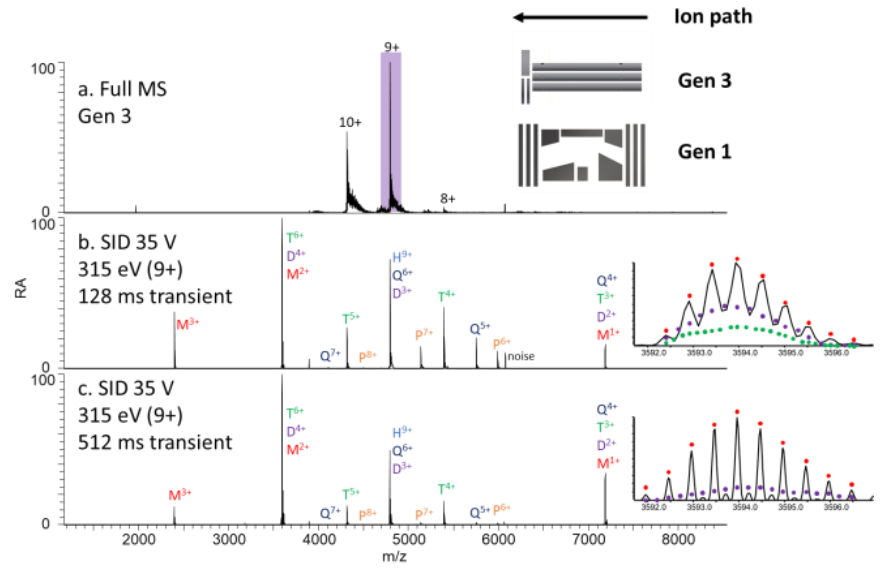

Figure 6: Surface-induced dissociation using a Gen 3 device on an EMR Orbitrap platform: (a) full scan mass spectrum of HFQ65 charge reduced using TEAA, (b) SID (315 eV) of the 9+ precursor using the 35k resolution setting (128 ms transient), and (c) SID (315 eV) of the 9+ precursor using the 140k resolution setting (512 ms transient). Insets show the peak at $m / z$ 3594. A bias against the larger oligomers is observed at longer transient lengths due to the increased path length. A comparison is given to the FTICR SID spectra of the 10+ charge state with different transient lengths in Figure S17. 


\section{Simple and minimally invasive SID devices for native mass spectrometry}

Dalton T. Snyder ${ }^{1}$; Erin Panczyk ${ }^{1,2}$; Arpad Somogyi ${ }^{1,3}$; Desmond Kaplan ${ }^{4}$; Vicki Wysocki*1-3

${ }^{1}$ Resource for Native MS Guided Structural Biology

${ }^{2}$ Department of Chemistry and Biochemistry

${ }^{3}$ Campus Chemical Instrument Center, Mass Spectrometry and Proteomics

The Ohio State University, Columbus OH, USA 43210

${ }^{4}$ KapScience LLC, Tewksbury, MA, USA 01876

*Corresponding author: wysocki.11@osu.edu

\section{Contents}

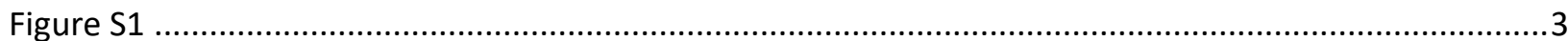

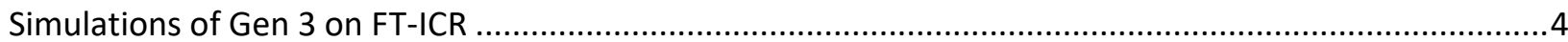

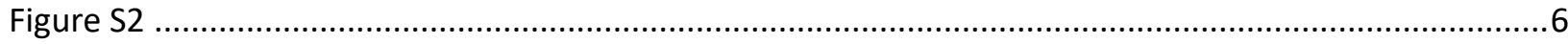

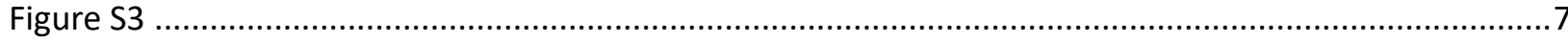

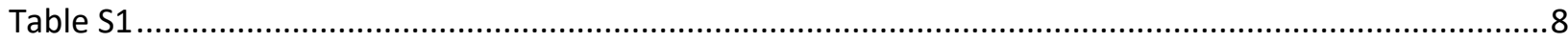

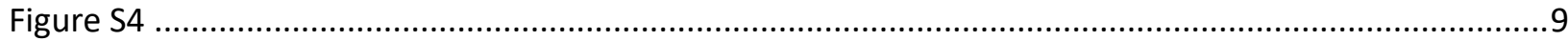

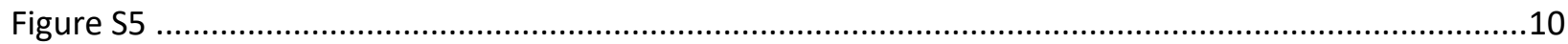

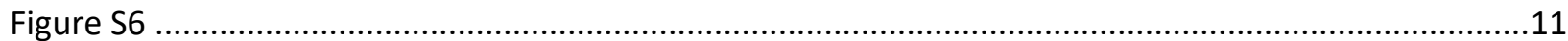

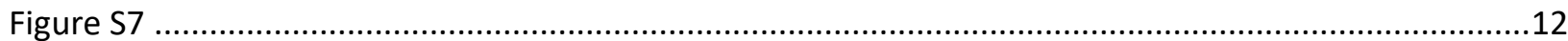

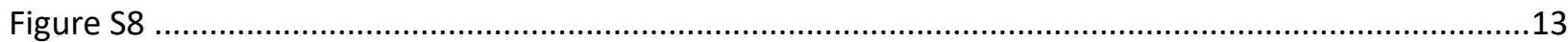

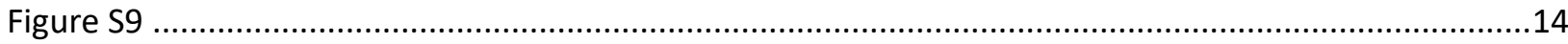

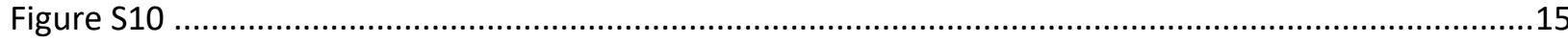

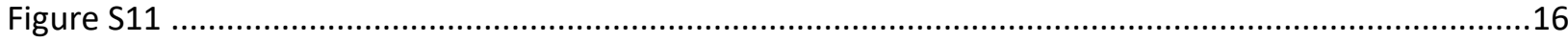

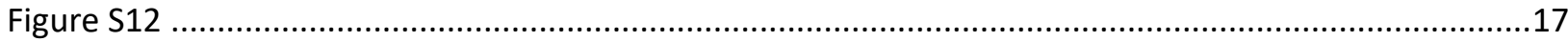

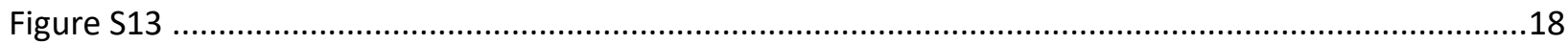

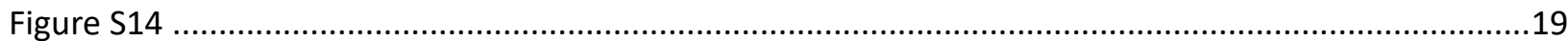

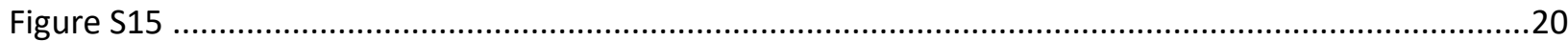

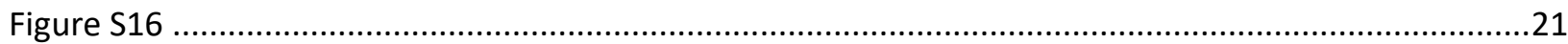

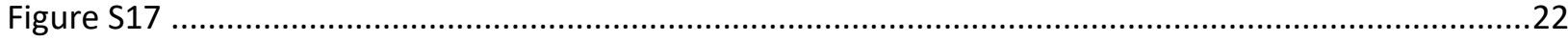

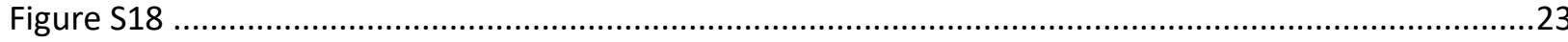




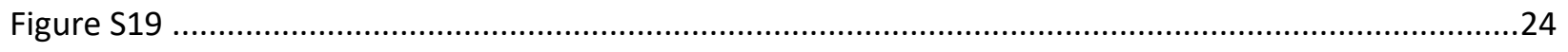

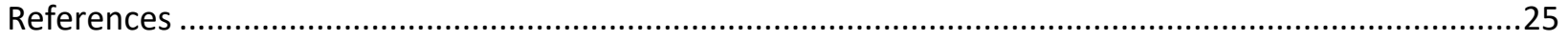


a

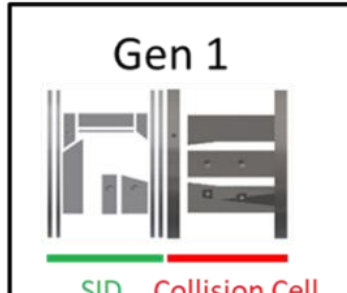

SID Collision Cell

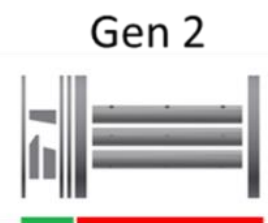

SID Collision Cell

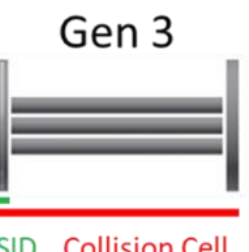

b

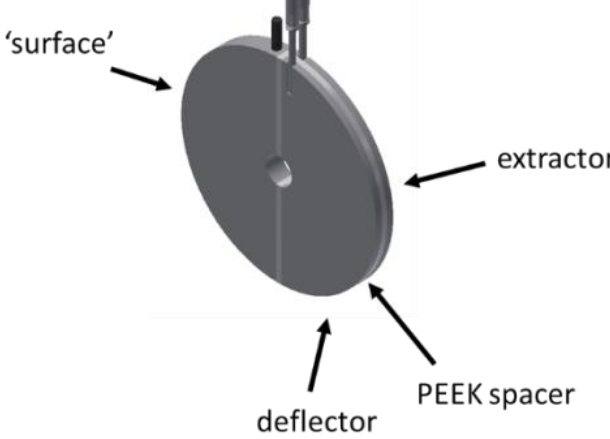

d

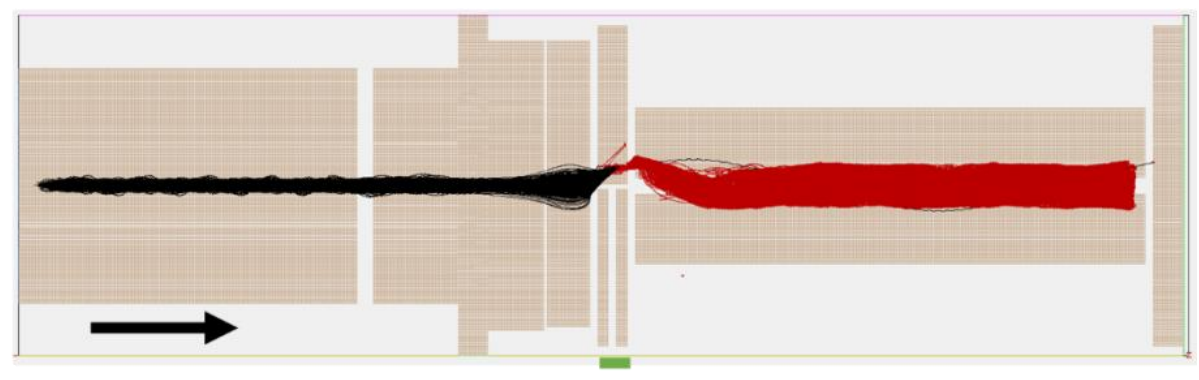

$3.25 \mathrm{~mm}$ c

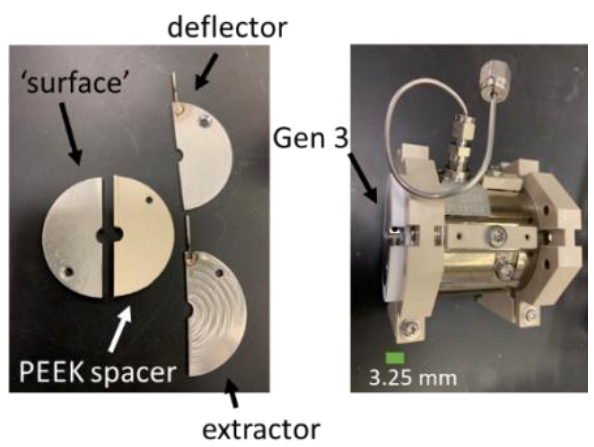

extractor

Figure S1: (a) Evolution of surface-induced dissociation devices in a 15 T FT-ICR mass spectrometer. In the first two generations, the device consists of two distinct regions (total length 62 $\mathrm{mm}$ ), one for SID (green bar) and one for CID and ion accumulation (red bar). In Gen 3 on the FT-ICR, SID is performed in the front endcap electrode of the collision cell by implementing a double split lens design. The Gen 3 SID device, shown as a 3D drawing in (b) and a picture of the fabricated device in (c), consists of three electrodes and a PEEK spacer and is small enough (3.25 mm length) to replace the endcap of a commercial collision cell (c, right). (d) Simulations (view from top) of 100 ions in the Gen 3 device (53 $\mathrm{kDa}, 10+$ ) indicate high collection efficiency after surface collision (SID $85 \mathrm{~V}$ ) due to the presence of a trapping rf field directly behind the surface without any intermediate lenses. 


\section{Simulations of Gen 3 on FT-ICR}

Initial simulations of Gen 3 on the FT-ICR were carried out in SIMION 8.1 (Scientific Instrument Services, Ringoes, NJ, USA) and consisted of the quadrupole mass filter (with pre- and post-filters), two focusing lenses after the post-filter, and the Bruker collision cell with Gen 3 replacing the front endcap. The initial conditions of the ions were as follows: number of ions, 1,000; ion mass, $53.22 \mathrm{kDa}$; ion charge, 10+; source position, Gaussian distribution with $0.2 \mathrm{~mm}$ standard deviation in the radial dimension; initial ion kinetic energy, Gaussian distribution with 25 +/- 5 (FWHM) eV; time-of-birth, uniform distribution between 0 and 5 microseconds. The quadrupole was operated in rf-only mode $(2,000 \mathrm{Vpp}$ at $880 \mathrm{kHz}$ ) to transmit the ions to the surface where SID was simulated. To perform SID, the ion was stopped within $\sim 0.2 \mathrm{~mm}$ of the surface and its kinetic energy was reduced to retain $\sim 5 \%$ of its precollision kinetic energy. The ion's trajectory was then reflected in a specular manner with respect to the surface normal. Black and red traces show the ion trajectory before and after collision (Figure S1d shows SID $85 \mathrm{~V}$ of +10 streptavidin tetramer). Note that the mass of the ion does not change in this simulation because it is assumed that the ion fragments later in the collision cell, i.e. a shattering mechanism is unlikely for protein complexes (we will show evidence for this assumption later). The LUA program for simulating $\mathrm{dc}$ and $\mathrm{rf}$ ion optics as well as SID is the same as the one used in two recently published papers, ${ }^{1,2}$ though the Bruker collision cell is simulated in this work instead of the Gen 2 collision cell on the FT-ICR.

As shown in Figure S3, a 98.2\% ion survival was observed with Gen 3 compared to $80.8 \%$ survival in Gen 2 under comparable simulation conditions. Note that this result does not imply that nearly $100 \%$ of precursor ions should be successfully trapped experimentally after recoil off the surface. Rather, the implication is that relative to Gen 2, Gen 3 should have higher sensitivity. We then simulated less ideal conditions, i.e. a larger ion beam, greater initial kinetic energy dispersion, greater variability in reflection angle during SID, and variability in kinetic energy retention after surface collision. The results are collected in Table S1. In all cases, Gen 3 returns superior survival results. Gen 3 also appears more tolerant to heterogeneous ion beams than Gen 2. For example, increasing the range of possible reflection angles from specular $+/-0^{\circ}$ to specular $+/-20^{\circ}$ causes a drop in survival from $80.8 \%$ to $73.6 \%$ for Gen 2, whereas virtually no difference is observed for Gen 3 because the ions proceed directly into a tolerant rf field after collision. Both devices are particularly sensitive to ion beam size, with survivals of $80.8 \%$ and $54.7 \%$ (Gen 3 and 2, respectively) when the ion beam standard deviation in the radial dimension is doubled. Gen 3 appears more tolerant to kinetic energy retention after surface collision, with $95.8 \%$ survival compared to Gen 2's $39.3 \%$ survival if the kinetic energy retention upon collision is a range of values ( $5 \%+/-2 \%$ of the ion's initial kinetic energy) rather than a fixed value $(5 \%)$. The large difference observed in this particular simulation can be rationalized by considering that the DC ion optics after surface collision are not tolerant to kinetic energy heterogeneity, whereas an rf potential can efficiently trap ions over a wide range of kinetic energies. These results suggest that the performance of Gen 3 compared to Gen 2 should be most improved for large and highly charged ions, which tend to have greater beam heterogeneity. As we will show later, experimental results on the FTICR were in agreement with this observation.

So why is the device design of Gen 3 more tolerant to beam heterogeneity? First, after surface collision, the ions are extracted directly into a trapping rf field. Unlike Gen 2 and Gen 1, there are no intermediate electrodes (between surface and collision cell) on which ions can neutralize after they are reflected off the surface. This is especially important for ion beams that are large or diverse in terms of 
radial position and kinetic energy. Second, all previous device designs in our laboratory have used flat surfaces. ${ }^{1-6}$ In contrast, Gen 3 uses a cylindrical surface. When a converging or diverging ion beam hits a flat surface, in both cases the reflected ion beam diverges because variations in angle of approach translate directly into variations in reflection angle. For the half-cylindrical surface of Gen 3, no matter where on the surface the ions hit, they are always focused towards the radial center of the collision cell. 


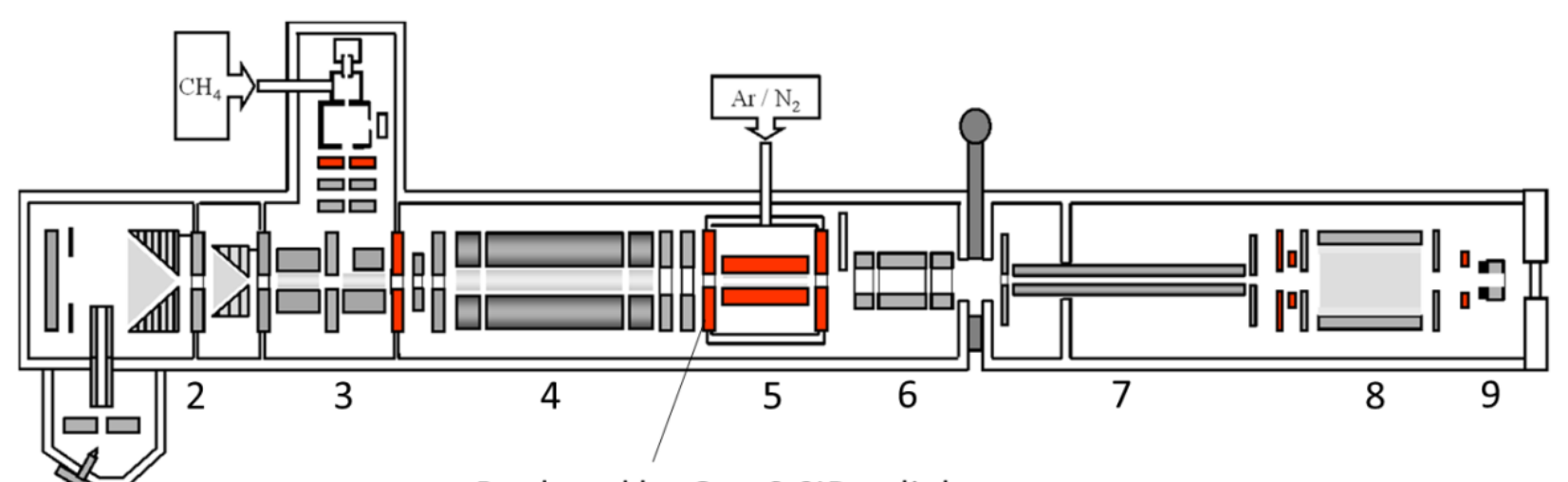

Replaced by Gen 3 SID split lens

Figure S2: Instrument diagram for Bruker SolariX XR 15 T FT-ICR mass spectrometer modified for SID. Important elements are labeled: 1 . nESI/ESI source; 2 . Dual ion funnel; 3. Transport multipole with ETD reagent manifold; 4. Quadrupole mass filter (up to $\mathrm{m} / \mathrm{z}$ 6,000); 5 . Collision cell; 6 . Focusing lenses; 7. Transport multipole; 8. FT-ICR cell; 9. ECD apparatus. In this work a Gen 3 split lens capable of SID replaced the front endcap, as indicated. 

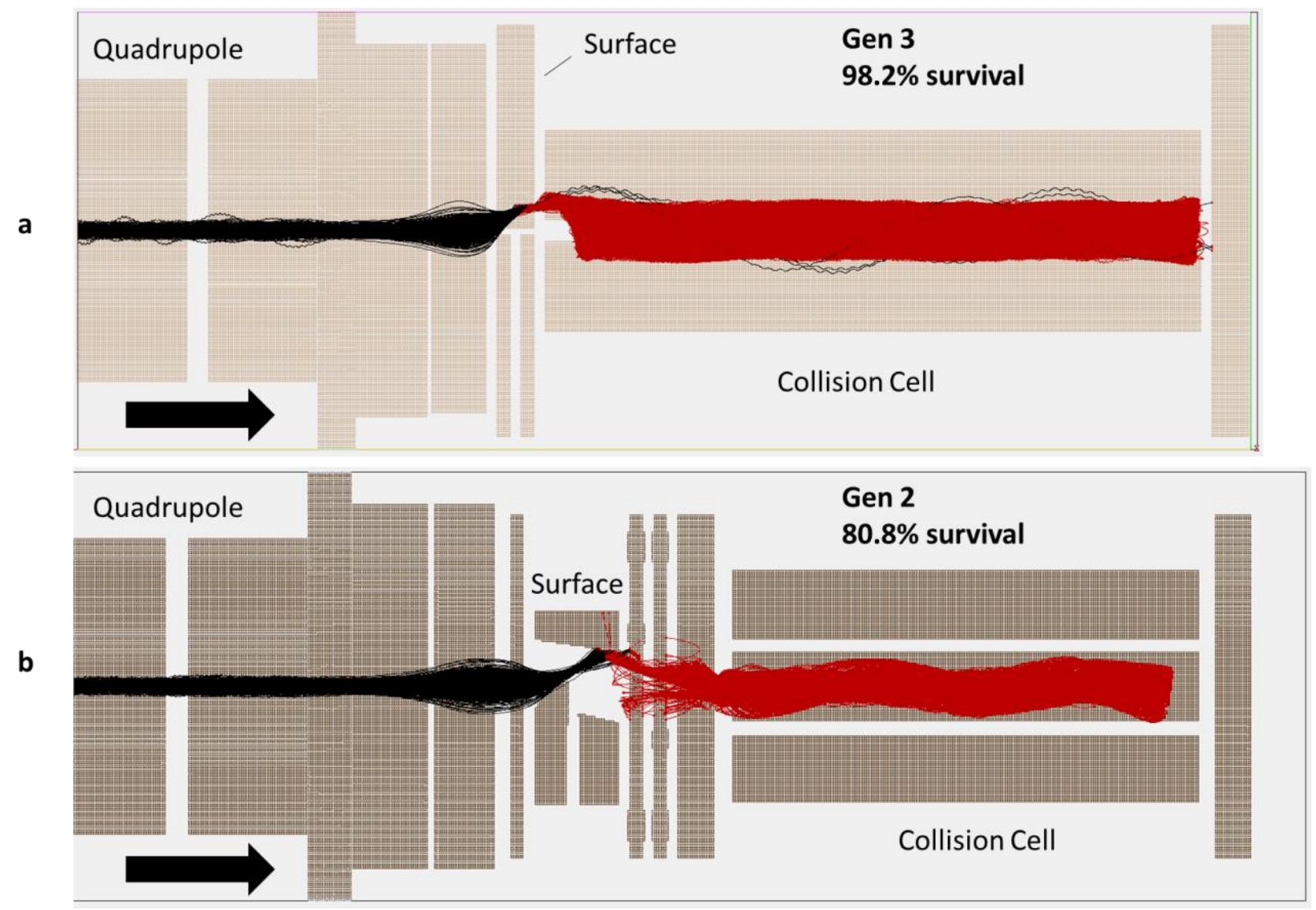

Figure S3: SIMION 8.1 Simulations of surface-induced dissociation (acceleration potential of $85 \mathrm{~V}$ ) using (a) Gen 3 and (b) Gen 2 under 'ideal' conditions. Parameters were as follows: number of ions, 1,000; ion mass, $53.22 \mathrm{kDa}$; ion charge, 10+; source position, Gaussian distribution with $0.2 \mathrm{~mm}$ standard deviation in the radial dimension; initial ion kinetic energy, Gaussian distribution with 25 +/- 5 (FWHM) eV; timeof-birth, uniform distribution between 0 and 5 microseconds; ion kinetic energy retention after surface collision, $5 \%+/-0 \%$; reflection angle, specular $+/-0^{\circ}$. The ion mass and charge after surface collision did not change during the simulation because the ion does not fragment until it is trapped in the collision cell. Survival percentages indicate which ions were successfully trapped in the collision cell (i.e. ions that did not splat on any electrode during the simulation). 
Table S1: Comparison between survival of ions after SID in Gen 3 and Gen 2 under various conditions. The conditions were the same as described in Figure $\mathbf{S 3}$ aside from the changes noted in the 'Conditions' column.

\begin{tabular}{|l|c|c|}
\hline Conditions & Gen 3 Survival & Gen 2 Survival \\
\hline 'Ideal' (conditions from Figure S3) & $98.2 \%$ & $80.8 \%$ \\
\hline Reflection angle, specular +/- 20 ${ }^{\circ}$ instead of +/- $0^{\circ}$ & $98.2 \%$ & $73.6 \%$ \\
\hline Initial ion KE FWHM of $10 \mathrm{eV}$ instead of $5 \mathrm{eV}$ & $98.3 \%$ & $76.0 \%$ \\
\hline $\begin{array}{l}\text { Source position: Gaussian distribution with 0.4 mm standard } \\
\text { deviation instead of 0.2 mm (i.e. a larger ion beam) }\end{array}$ & $80.8 \%$ & $54.7 \%$ \\
\hline $\begin{array}{l}\text { Greater variability in ion KE retention after surface collision, } \\
5 \%+/-2 \% \text { instead of 5\% +/- 0\% }\end{array}$ & $95.8 \%$ & $39.3 \%$ \\
\hline
\end{tabular}



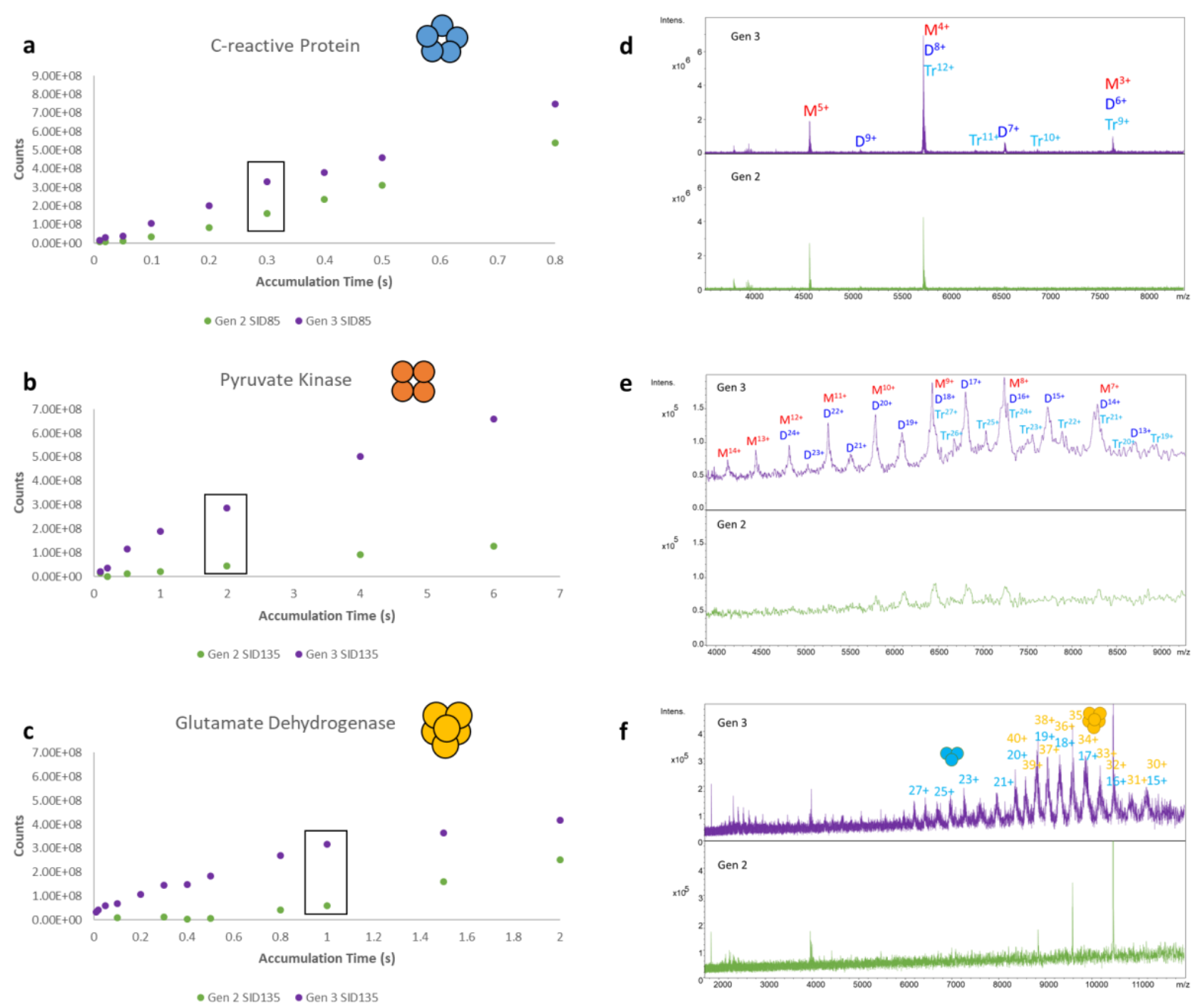

Figure S4: Comparison of Gen 2 and Gen 3 SID sensitivity as a function of accumulation time for (a) charge reduced C-reactive protein (SID $85 \mathrm{~V}$ ), (b) pyruvate kinase (SID $135 \mathrm{~V}$, spectrum smoothed using a 2 Da wide Gaussian filter), and (c) glutamate dehydrogenase (SID $135 \mathrm{~V}$ ). No isolation was performed in this experiment on the FT-ICR. The average charge state of the precursors was (a) +18 , (b) +35 , and (c) +40. Panels (d), (e), and ( $f$ ) show corresponding spectra for the data points highlighted by the black boxes. $\mathrm{M}=$ monomer, $\mathrm{D}=$ dimer, $\mathrm{T}=$ trimer 

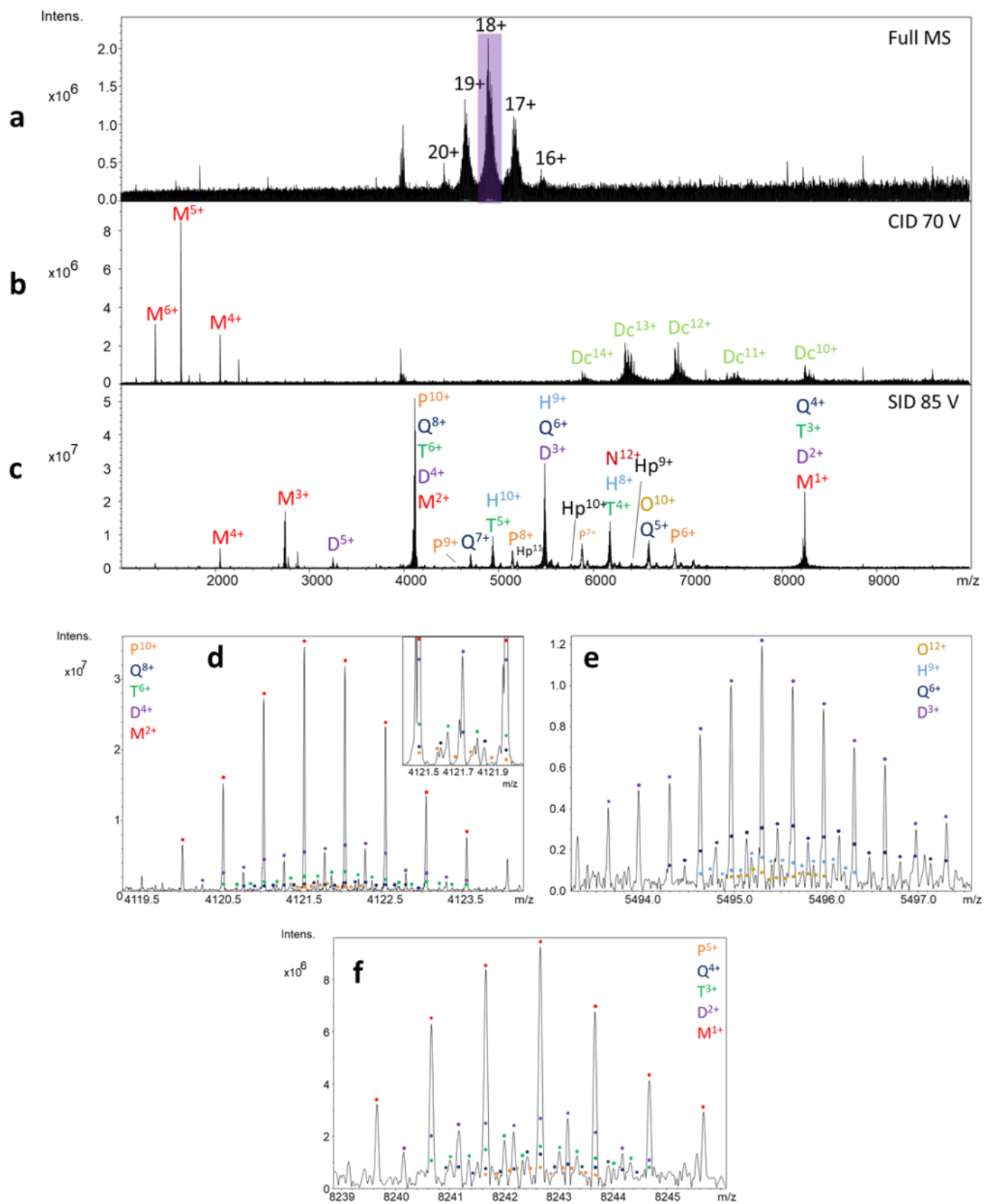

Figure S5: Native MS of homo-11mer TRAP (in 200 mM EDDA with 14 equivalents of trp) on the FT-ICR: (a) full scan, (b) CID $70 \mathrm{~V}$ of the $18+$ charge state, (c), SID $85 \mathrm{~V}$ of the $18+$ charge state, and (d-f) expanded views of overlapping peaks but obtained at higher resolution ( $9 \mathrm{~s}$ transient vs $2.2 \mathrm{~s}$ transient). $\mathrm{M}=$ monomer, $\mathrm{D}=$ dimer, $\mathrm{T}=$ trimer, $\mathrm{Q}=$ tetramer, $\mathrm{P}=$ pentamer, $\mathrm{H}=$ hexamer, $\mathrm{Hp}=$ heptamer, $\mathrm{O}=$ octamer, $\mathrm{N}=$ nonamer, $\mathrm{Dc}=$ decamer 
a
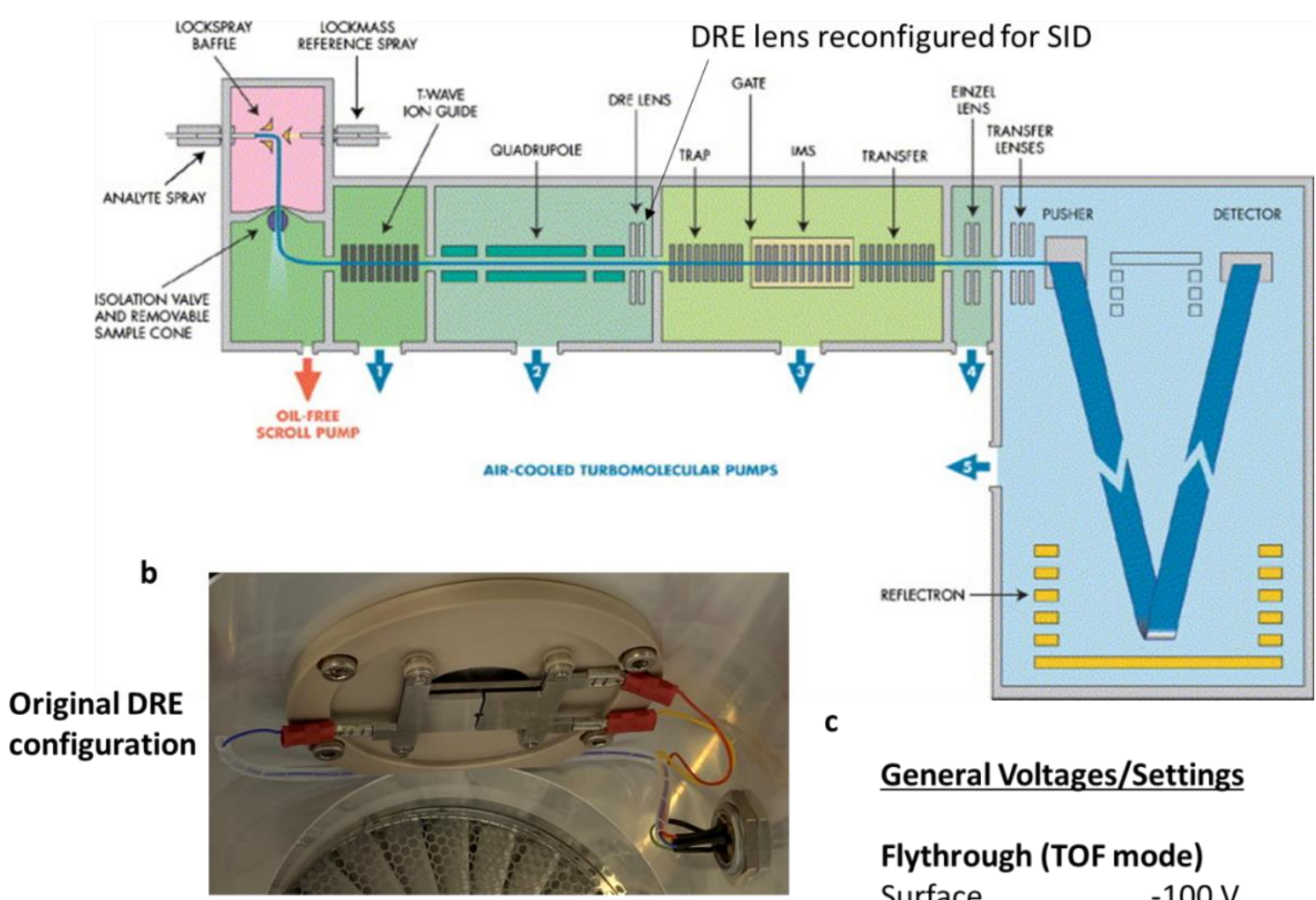

C

General Voltages/Settings

Flythrough (TOF mode)

Surface $\quad-100 \mathrm{~V}$

Deflector $\quad-100 \mathrm{~V}$

Extractor $\quad-100 \mathrm{~V}$

Trap CE 4

SID 85 V (TOF mode)

Surface $-100 \mathrm{~V}$

Deflector $\quad-60 \mathrm{~V}$

Extractor $\quad-100 \mathrm{~V}$

Trap CE 85

Figure S6: Surface-induced dissociation in a Synapt G2 using a split lens design. (a) Synapt G2 instrument schematic with arrow indicating former DRE (dynamic range enhancement) lens reconfigured for SID as shown in panel (b). The Gen 3 SID configuration consists of a surface fabricated in-house with the split lens from the original DRE configuration moved to the same side of the ion path in order to function as deflector and extractor for SID. Typical voltages for transmission (flythrough) and SID modes are given in (c) for the case of TOF mode (no ion mobility) on the G1. The DRE lens is identical in the Synapt G2 design. Panel (a) adapted from Pringle, S. D.; Giles, K.; Wildgoose, J. L.; Williams, J. P.; Slade, S. E.; Thalassinoas, K.; Bateman, R. H.; Bowers, M. T.; Scrivens, J. H. Int. J. Mass Spectrom. 2007, 261, 1-12. Copyright 2007, with permission from Elsevier. 


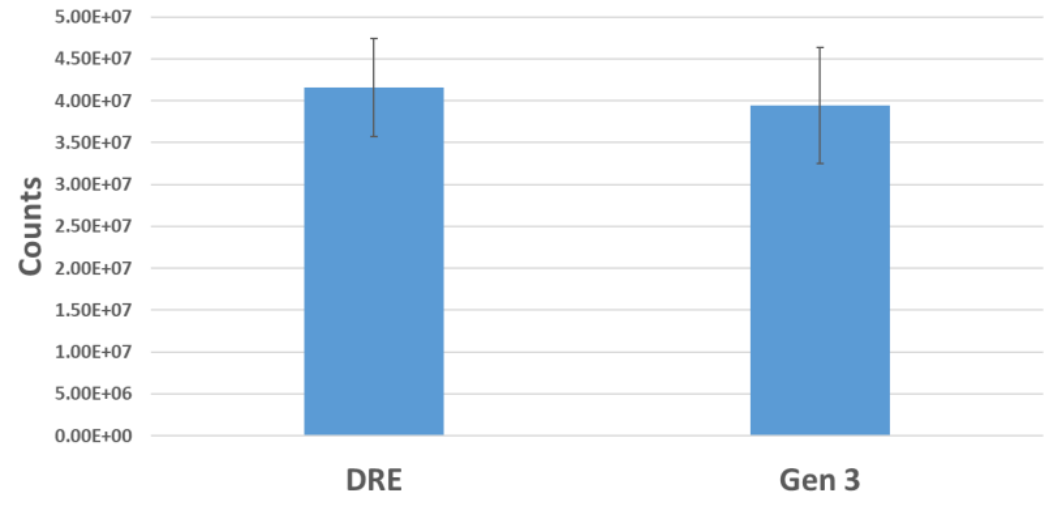

Figure S7: Flythrough (IM-TOF mode) intensities of $115 \mathrm{kDa}$ pentamer C-reactive protein using the original DRE lens of a Synapt G2 and Gen 3 installed in place of the DRE lens. No statistically significant difference (over 3 replicate $n E S I$ emitters) was observed when the DRE lens was reconfigured. 
a

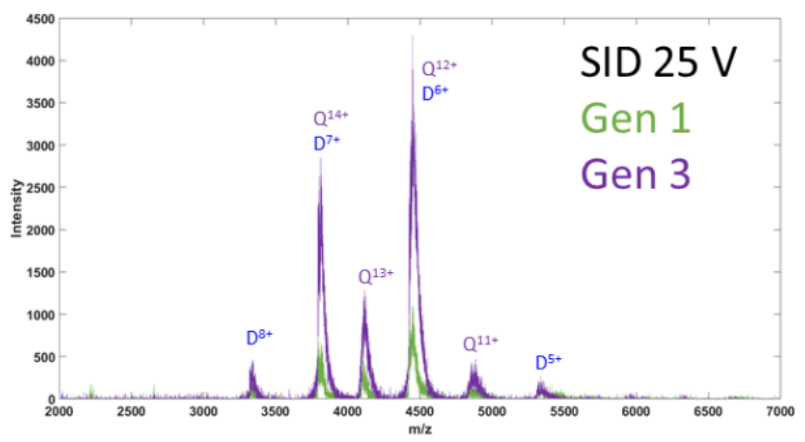

b

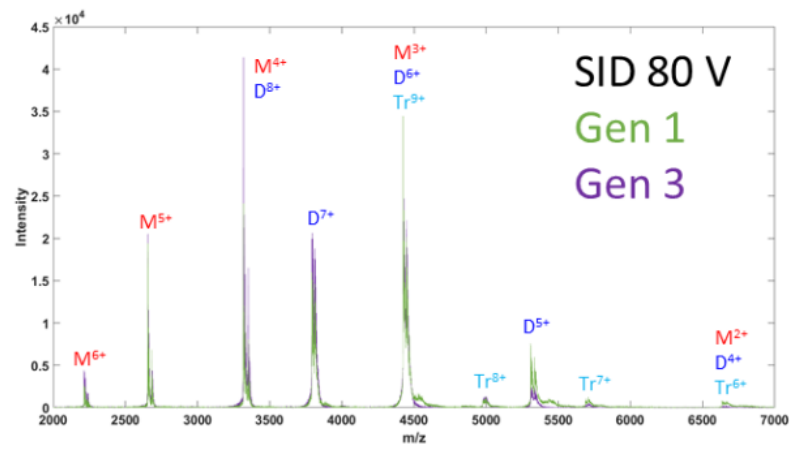

C

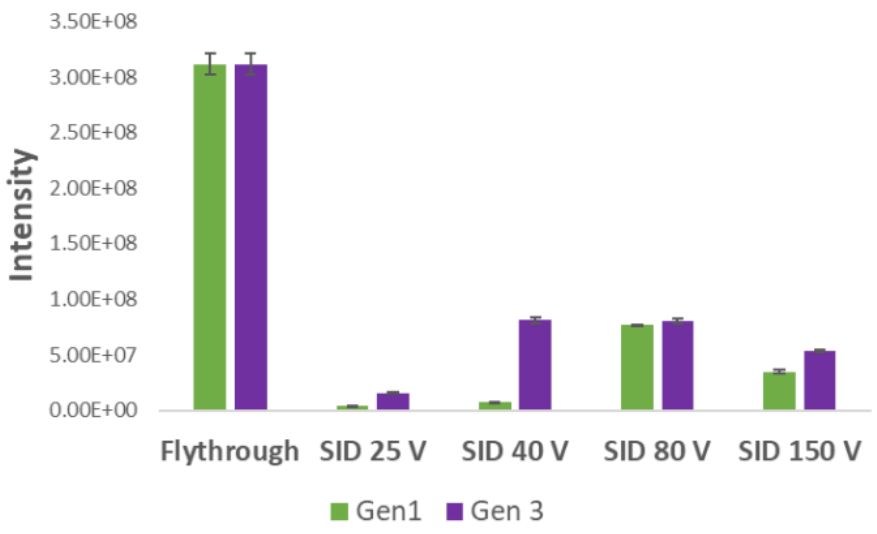

Figure S8: Comparison of SID spectra and signal intensities for streptavidin in EDDA using Gen 1 and Gen 3 on the G2: (a) Surface-induced dissociation with $25 \mathrm{~V}$ potential difference (no isolation), (b) surfaceinduced dissociation with $80 \mathrm{~V}$ potential difference (no isolation) and (c) corresponding total ion counts at various SID energies (three technical replicate each). 


\section{a}

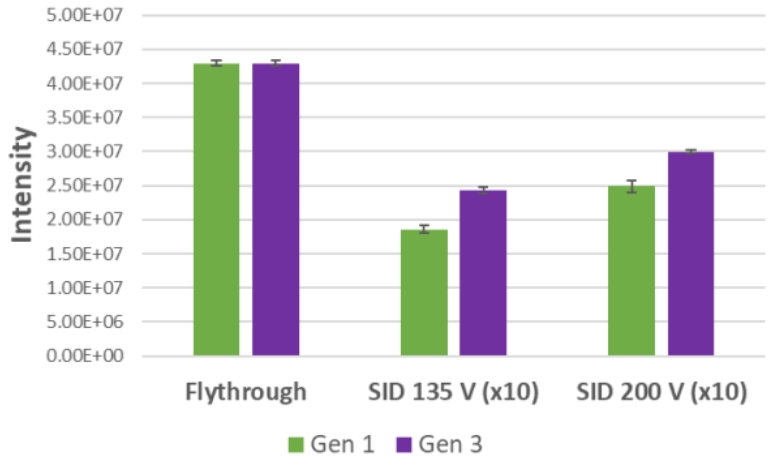

b

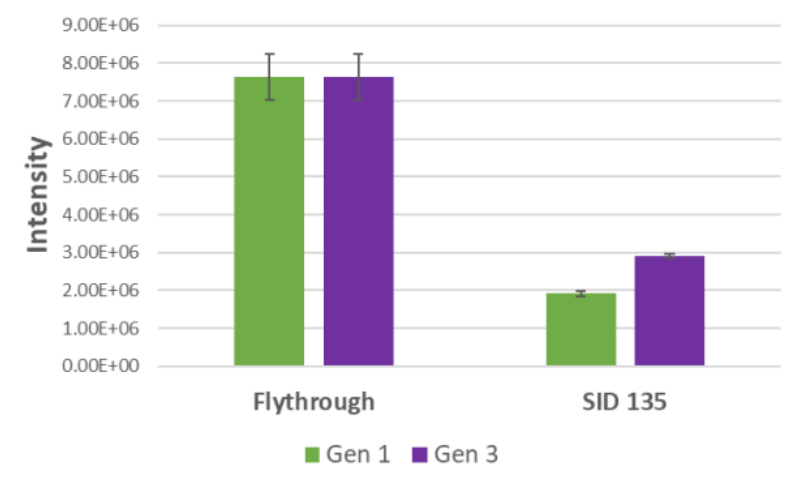

Figure S9: Comparison of signal intensities in flythrough and SID modes for (a) pyruvate kinase in EDDA and (b) glutamate dehydrogenase. 


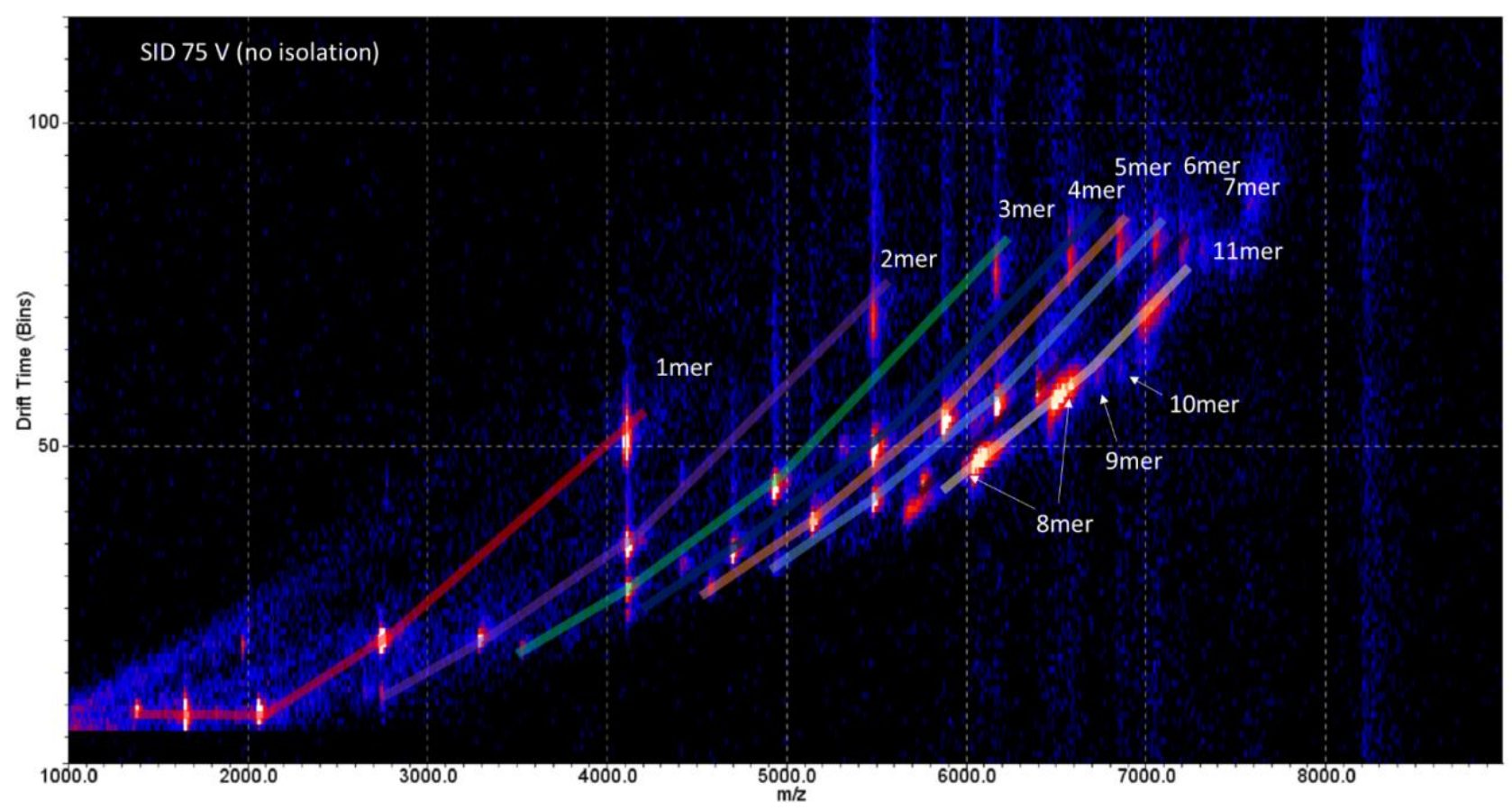

Figure S10: SID-IM of 11mer apoTRAP in EDDA on a Synapt G1 (without quadrupole isolation) using a Gen 3 SID device. Because the subunits are arranged in a ring, all oligomeric fragments are observed. 
a

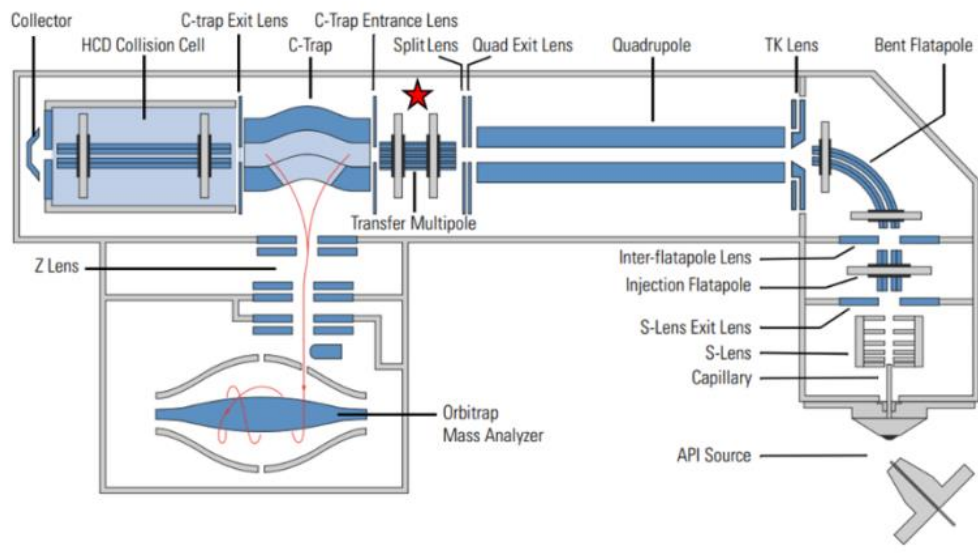

b

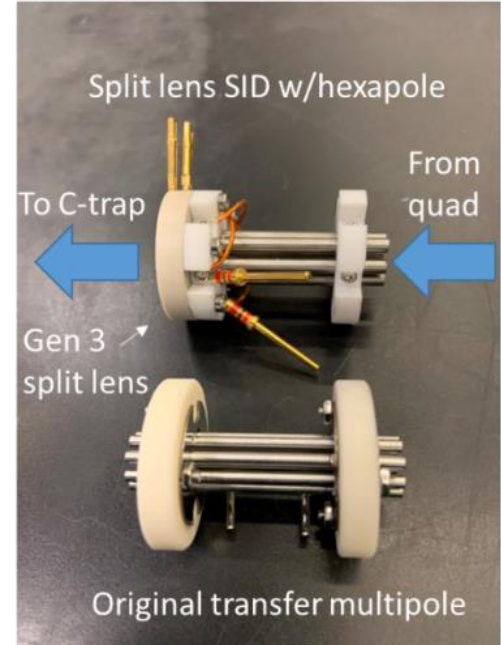

Figure S11: Implementation of Gen 3 SID device in an extended mass range (EMR) Orbitrap Exactive. (a) Schematic of EMR Orbitrap with red star indicating transfer multipole that was replaced by the Gen 3 SID device shown in (b). The SID device consists of a transfer hexapole and a doubly split lens similar to the Gen 3 designs on the FT-ICR and Synapt. The hexapole has length $39.00 \mathrm{~mm}$, inscribed diameter (distance between opposite rods) of $8.75 \mathrm{~mm}$, and rod diameter of $4.75 \mathrm{~mm}$. The split lens, consisting of deflector, surface, and extractor, has inner aperture diameter of $2.00 \mathrm{~mm}$ and total thickness of 3.00 $\mathrm{mm}$. The electrodes are made of stainless steel, the split lens holder is made of PEEK, and the hexapole holder is made of ceramic Macor. The rf for the hexapole is supplied by the same driver that supplied the $r f$ for the original transfer multipole. 


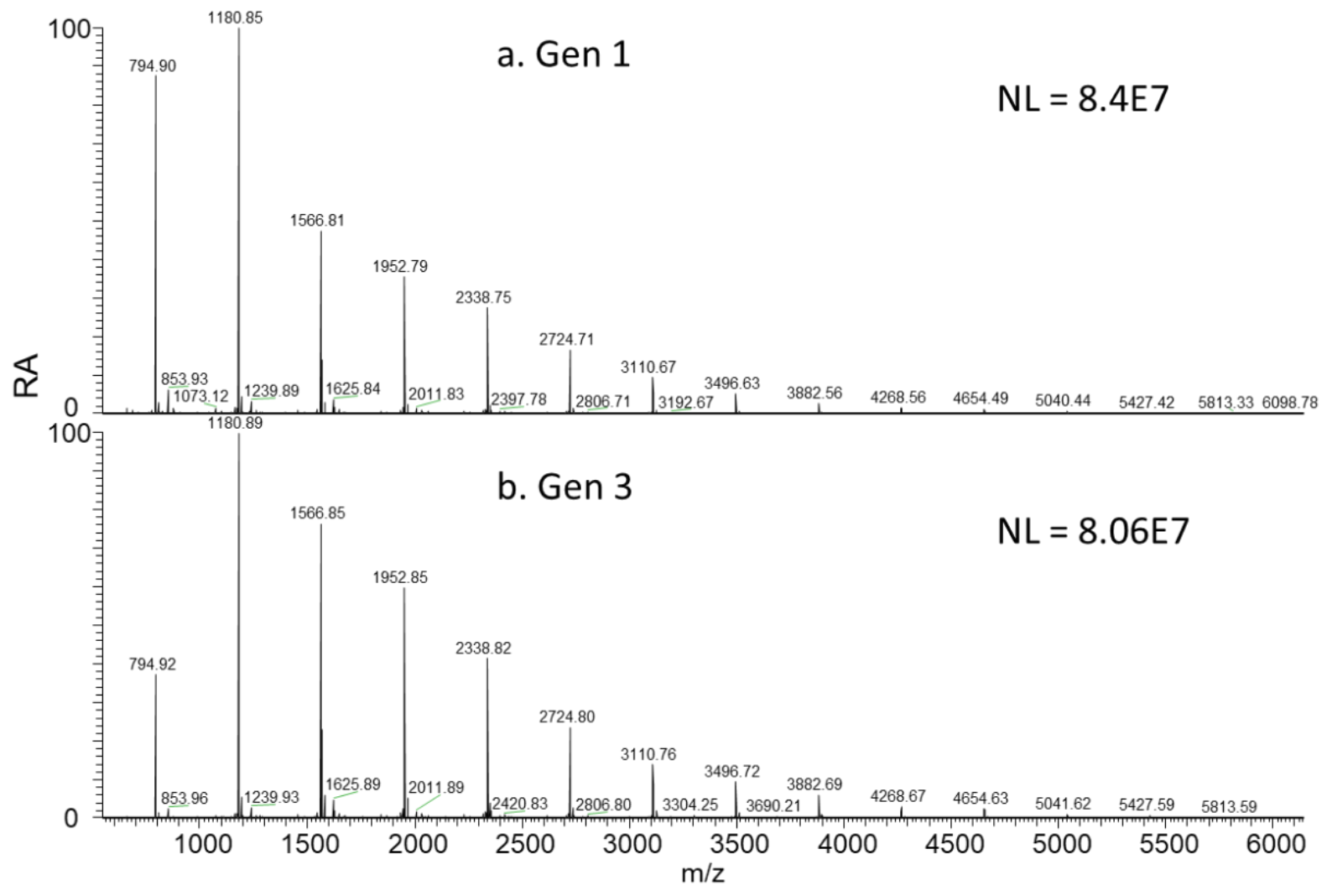

Figure S12: Transmission of CsPFHA clusters on the EMR using (a) Gen 1 and (b) Gen 3. Small differences in relative transmission can be attributed to the mass biases of the rf-only hexapole of Gen 3 compared to the dc-only optics of Gen 1 . NL = normalization level 


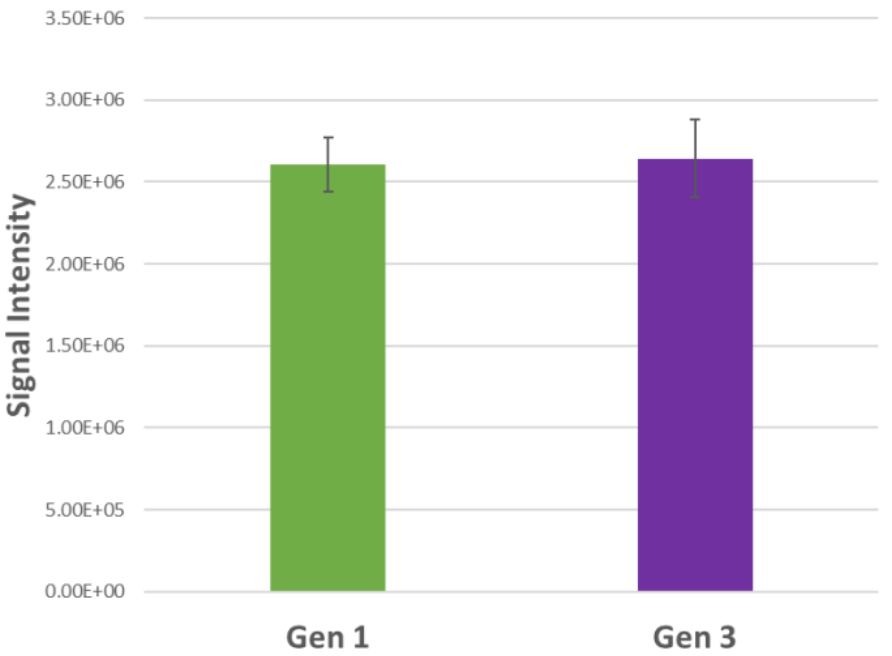

Figure S13: Transmission of $330 \mathrm{kDa}$ hexamer glutamate dehydrogenase using Gen 1 and Gen 3 on the EMR. Over three replicate nESI tips, no differences in intensity were observed. 


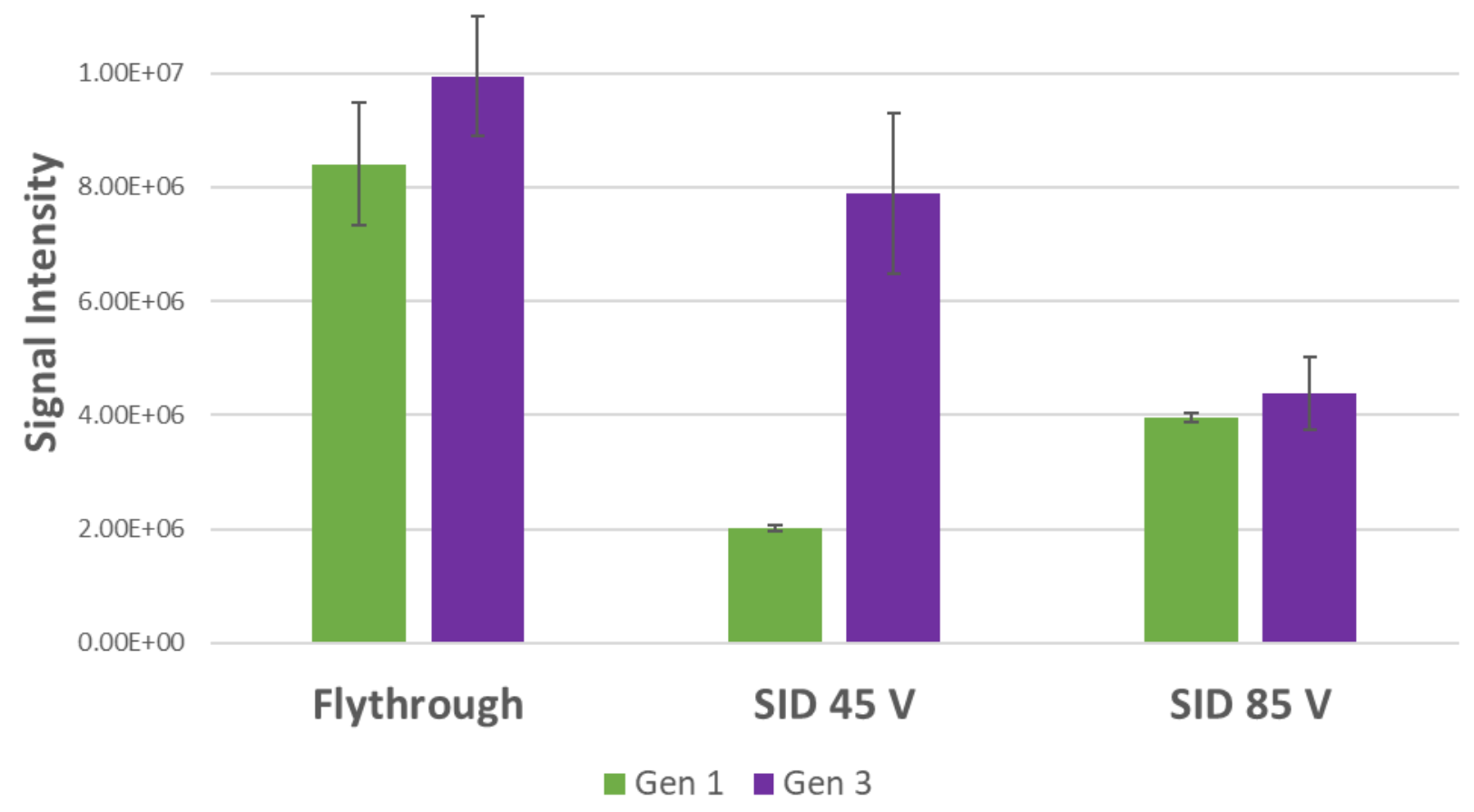

Figure S14: Comparison of signal intensity for 53 kDa streptavidin tetramer using Gen 1 and Gen 3 on the EMR. A difference in signal intensity is noted only for the low energy regime (SID $45 \mathrm{~V}$ ). Higher energies were not tested. 


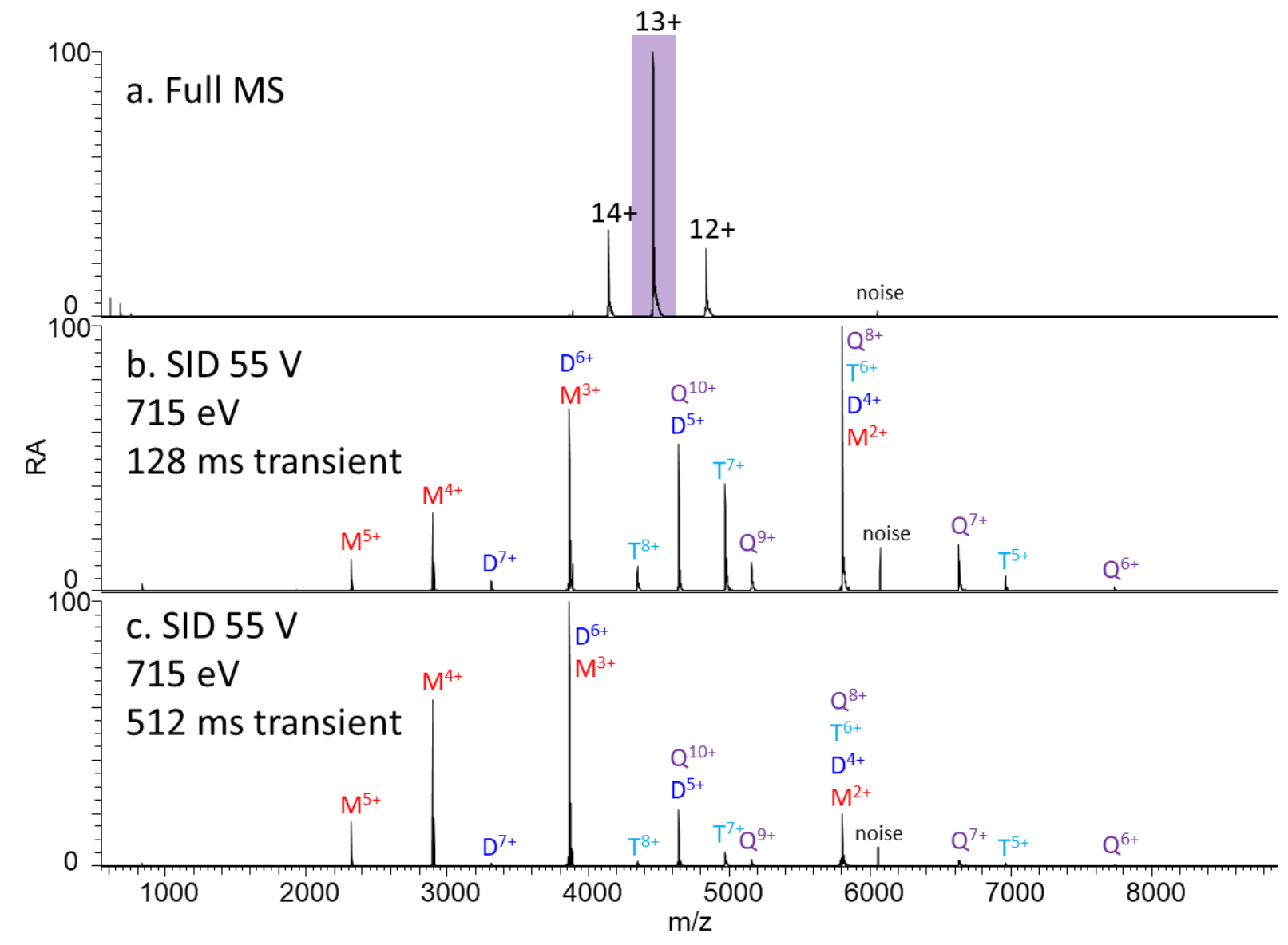

Figure S15: Surface-induced dissociation of $58 \mathrm{kDa}$ pentamer cholera toxin B (CTB) using Gen 3 on the EMR Orbitrap. (a) Full scan mass spectrum, (b) surface-induced dissociation spectrum (715 eV) of the $13+$ charge state at $35 \mathrm{k}$ resolution setting (128 ms transient), and (c) SID spectrum (715 eV) of the 13+ charge state at $140 \mathrm{k}$ resolution setting ( $512 \mathrm{~ms}$ transient). A substantial bias against the higher order oligomers is observed with increased transient lengths. 

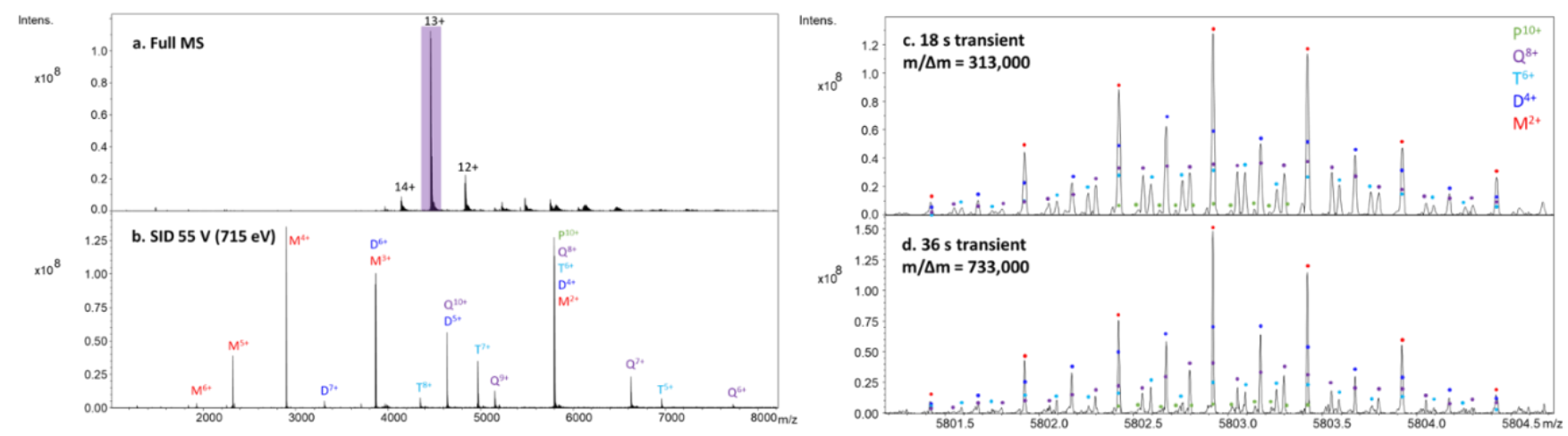

Figure S16: Surface-induced dissociation of $58 \mathrm{kDa}$ pentamer cholera toxin B (CTB) using Gen 3 on the FT-ICR. (a) Full scan mass spectrum, (b) surface-induced dissociation spectrum (715 eV) of the 13+ charge state using an $18 \mathrm{~s}$ transient, and $(\mathrm{c}, \mathrm{d})$ zoom in of the overlapping fragments in the SID spectrum $(715 \mathrm{eV})$ of the $13+$ charge state using different transient lengths. Much less bias is observed on the FTICR compared to the EMR with increasing transient length. 


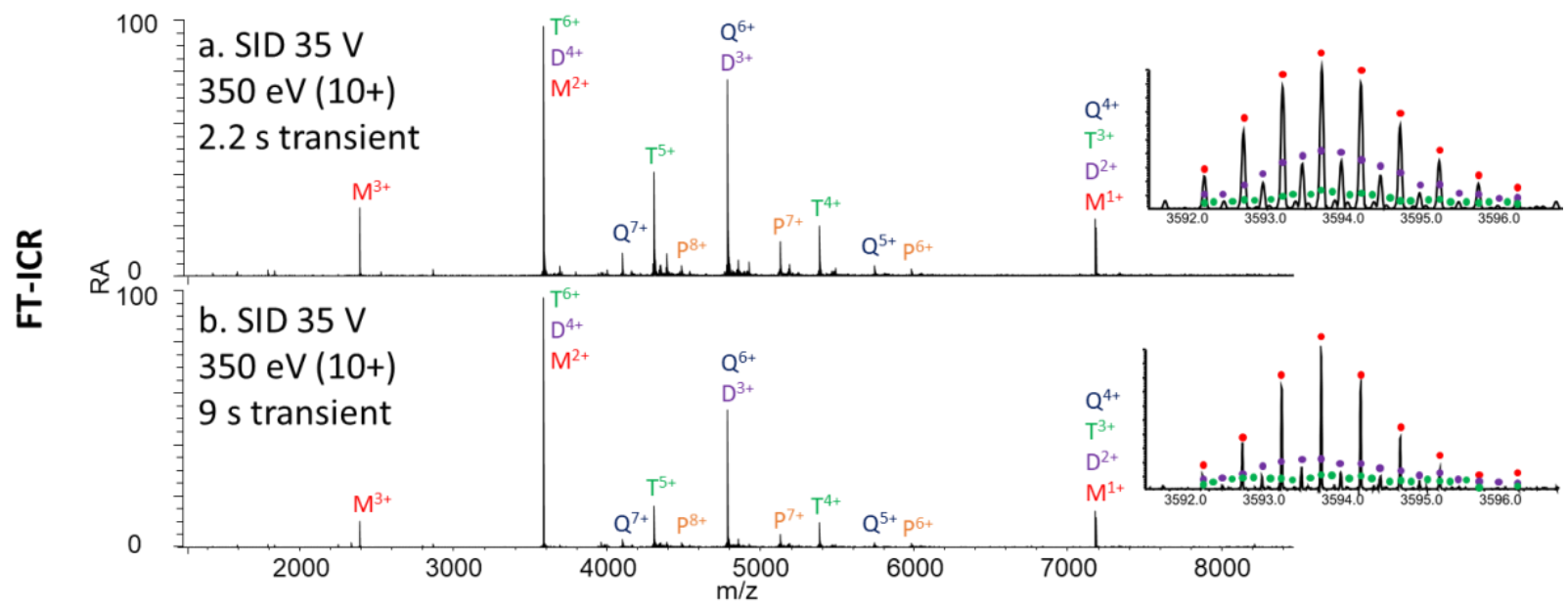

Figure S17: Surface-induced dissociation spectra of the 10+ charge state of homohexamer HFQ65 using different transient lengths of (a) $2.2 \mathrm{~s}$ and (b) $9 \mathrm{~s}$ on the FT-ICR.

22 


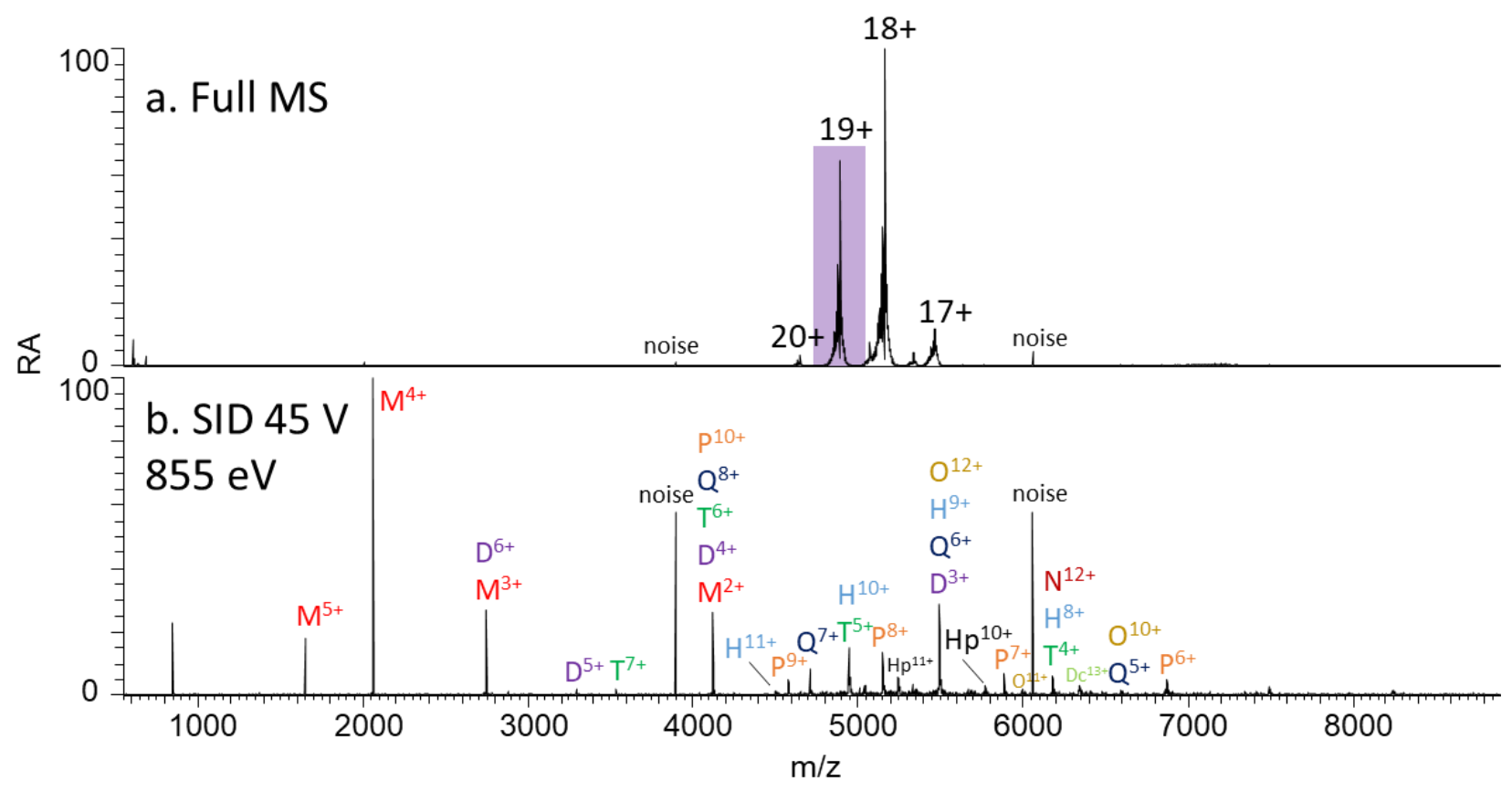

Figure S18: Surface-induced dissociation spectrum of 11mer holoTRAP protein complex using Gen 3 on the EMR Orbitrap. (a) Full scan mass spectrum and (b) SID spectrum of the 19+ charge state (855 eV). Resolution setting 35k (128 ms transient). 


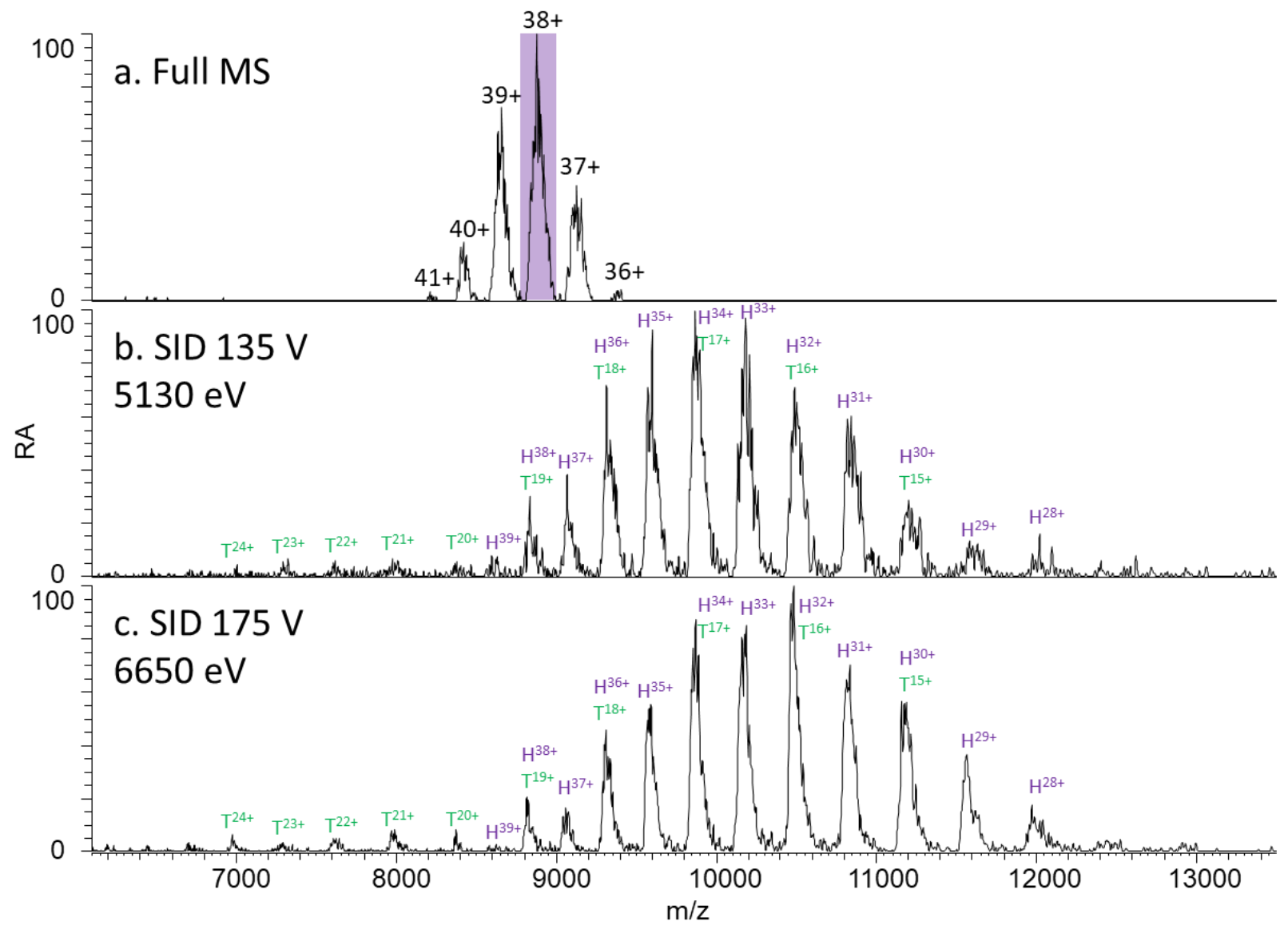

Figure S19: Surface-induced dissociation of $330 \mathrm{kDa}$ glutamate dehydrogenase hexamer using Gen 3 on the EMR Orbitrap. (a) Full scan mass spectrum and $(b, c)$ surface-induced dissociation spectra of the 38+ charge state at $5130 \mathrm{eV}$ and $6650 \mathrm{eV}$, respectively. Resolution setting 35k (128 ms transient). 


\section{References}

(1) Snyder, D. T.; Panczyk, E.; Stiving, A. Q.; Gilbert, J. D.; Somogyi, A.; Kaplan, D.; Wysocki, V. Design and Performance of a Second-Generation Surface-Induced Dissociation Cell for Fourier Transform Ion Cyclotron Resonance Mass Spectrometry of Native Protein Complexes. Anal. Chem. 2019, 91 (21), 14049-14057. https://doi.org/10.1021/acs.analchem.9b03746.

(2) Stiving, A. Q.; Gilbert, J. D.; Jones, B. J.; Wysocki, V. H. A Tilted Surface and Ion Carpet Array for SID. J. Am. Soc. Mass Spectrom. 2019. https://doi.org/10.1021/jasms.9b00009.

(3) Galhena, A. S.; Dagan, S.; Jones, C. M.; Beardsley, R. L.; Wysocki, V. H. Surface-Induced Dissociation of Peptides and Protein Complexes in a Quadrupole/Time-of-Flight Mass Spectrometer. Anal. Chem. 2008, 80 (5), 1425-1436. https://doi.org/10.1021/ac701782q.

(4) Zhou, M.; Huang, C.; Wysocki, V. H. Surface-Induced Dissociation of Ion Mobility-Separated Noncovalent Complexes in a Quadrupole/Time-of-Flight Mass Spectrometer. Anal. Chem. 2012, 84 (14), 6016-6023. https://doi.org/10.1021/ac300810u.

(5) VanAernum, Z. L.; Gilbert, J. D.; Belov, M. E.; Makarov, A. A.; Horning, S. R.; Wysocki, V. H. SurfaceInduced Dissociation of Noncovalent Protein Complexes in an Extended Mass Range Orbitrap Mass Spectrometer. Anal. Chem. 2019, 91 (5), 3611-3618. https://doi.org/10.1021/acs.analchem.8b05605.

(6) Yan, J.; Zhou, M.; Gilbert, J. D.; Wolff, J. J.; Somogyi, A.; Pedder, R. E.; Quintyn, R. S.; Morrison, L. J.; Easterling, M. L.; Pasa-Tolic, L.; et al. Surface-Induced Dissociation of Protein Complexes in a Hybrid Fourier Transform Ion Cyclotron Resonance Mass Spectrometer. Anal. Chem. 2017, 89 (1), 895901. https://doi.org/10.1021/acs.analchem.6b03986. 